\title{
Intrinsic Electromagnetic Variability In Celestial Objects Containing Rapidly Spinning Black Holes
}

\author{
Fan Zhang
}

\section{Digital Commons Citation}

Zhang, Fan, "Intrinsic Electromagnetic Variability In Celestial Objects Containing Rapidly Spinning Black Holes" (2016). Faculty \& Staff Scholarship. 15.

https://researchrepository.wvu.edu/faculty_publications/15

This Article is brought to you for free and open access by The Research Repository @ WVU. It has been accepted for inclusion in Faculty \& Staff Scholarship by an authorized administrator of The Research Repository @ WVU. For more information, please contact beau.smith@mail.wvu.edu. 


\title{
INTRINSIC ELECTROMAGNETIC VARIABILITY IN CELESTIAL OBJECTS CONTAINING RAPIDLY SPINNING BLACK HOLES
}

\author{
FAN ZHANG \\ Gravitational Wave and Cosmology Laboratory, Department of Astronomy, Beijing Normal University, Beijing 100875, China; fnzhang@bnu.edu.cn \\ Department of Physics and Astronomy, West Virginia University, P.O. Box 6315, Morgantown, WV 26506, USA \\ Received 2015 November 26; accepted 2015 December 27; published 2016 February 9
}

\begin{abstract}
Analytical studies have raised the concern that a mysterious expulsion of magnetic field lines by a rapidly spinning black hole (dubbed the black hole Meissner effect) would shut down the Blandford-Znajek process and quench the jets of active galactic nuclei and microquasars. This effect is, however, not seen observationally or in numerical simulations. Previous attempts at reconciling the predictions with observations have proposed several mechanisms to evade the Meissner effect. In this paper, we identify a new evasion mechanism and discuss its observational significance. Specifically, we show that the breakdown of stationarity is sufficient to remove the expulsion of the magnetic field at all multipole orders, and that the associated temporal variation is likely turbulent because of the existence of efficient mechanisms for sharing energy across different modes. Such an intrinsic (as opposed to being driven externally by, e.g., changes in the accretion rate) variability of the electromagnetic field can produce the recorded linear correlation between microvariability amplitudes and mean fluxes, help create magnetic randomness and seed sheared magnetic loops in jets, and lead to a better theoretical fit to the X-ray microvariability power spectral density.
\end{abstract}

Key words: galaxies: active - galaxies: jets - galaxies: magnetic fields

\section{INTRODUCTION}

Building on the work of Goldreich \& Julian (1969), Blandford \& Znajek (1977; BZ) proposed a mechanism that extracts the rotational energy of spinning black holes through a Penrose-like process, which has since become one of the most widely accepted contenders for explaining the energy source of outflowing jets seen in active galaxy nuclei (AGNs) containing supermassive black holes and microquasars containing stellar mass holes. For example, the measured jet power's dependence on the black hole spin is consistent with BZ predictions (Narayan \& McClintock 2012; Steiner et al. 2013). In order to operate effectively, the $\mathrm{BZ}$ mechanism requires the nearhorizon region to be sufficiently and appropriately magnetized. From the Penrose process point of view, the magnetic field lines should thread through the infinite redshift surface enclosing the ergosphere, while with the membrane paradigm description (Thorne et al. 1986) of the BZ process, one would need them to thread through the event horizon.

Astrophysically, the magnetic field surrounding black holes is expected to be a largely poloidal one confined by external matter (Begelman et al. 1984), which can be approximated as being asymptotically uniform when studying the vicinity of the black holes. The simplest and frequently invoked prototype solution for such a configuration is the Wald solution (Wald 1974) to the vacuum (without charge or current) Maxwell equations. When it is observed that as the black hole spin approaches extremality, the magnetic field lines of this solution are "expelled" from the horizon (see King et al. 1975; Bicak et al. 2006 and Figure 1), instead of penetrating it as in the case of slow spin. Concerns naturally arise that the fast-spinning black holes will not be adequately magnetized, so the BZ process shuts down, and the jets are quenched.

Although the complete expulsion of the field lines is only at extremality, the effect does bleed into the entire high-spin regime, causing a severe suppression of the jet power $P_{\mathrm{BZ}}$ from the $\mathrm{BZ}$ process, which is proportional to the quadrature of the magnetic flux across the horizon. Substituting in the Wald solution, we obtain $P_{\mathrm{BZ}}$ as a function of the dimensionless spin $a$ as (Bicak \& Janis 1985)

$$
\begin{aligned}
P_{\mathrm{BZ}} & =\frac{\pi B^{2} a^{2} r_{+}^{2}}{32 M^{2}}\left(1-\frac{a^{4}}{r_{+}^{4}}\right)^{2}, \\
\text { where } \quad r_{+} & =M+\sqrt{M^{2}-a^{2}},
\end{aligned}
$$

which is plotted in Figure 2. The $a$ appearing in the bracket is from the magnetic flux density contribution, and we see that power suppression sets in after $a \sim 0.8$, which is well within the possible astrophysical range. Theoretical computations by Thorne (1974) show that thin accretion disks can spin a black hole up to $a \sim 0.998$ (although the numerical experiment by Gammie et al. (2004) shows that lower equilibrium values at around 0.9 for particular magnetohydrodynamic (MHD) models are also possible). Within the BZ process, a nearextremal spin is also assumed when discussing the collimation of extracted energy into jets by an ion-supported torus (Rees et al. 1982; Begelman et al. 1984). Observationally, high spins have been reported for many systems, using a variety of measurement techniques. For example, the $\mathrm{Fe} \mathrm{K}$ line profile implies $a \approx 1$ for XTE J1650500 (Miller et al. 2002; Miniutti et al. 2004), while McClintock et al. (2006), Gou et al. (2011), and McClintock et al. (2013) have measured spins in excess of 0.95 for the primaries in black hole X-ray binaries Cygnus X-1 and GRS $1915+105$ through a continuum fitting method. Furthermore, when it comes to spin distribution among large populations, high spins are not rare. Summarizing X-ray reflection spectroscopy results, Reynolds (2013) concluded that a significant number of supermassive black holes have $a>0.9$. With a radiation efficiency study, Wang et al. (2006) estimated that the spins of essentially all black holes in quasars within the 


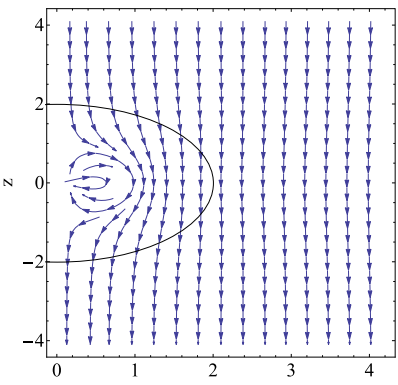

(a)

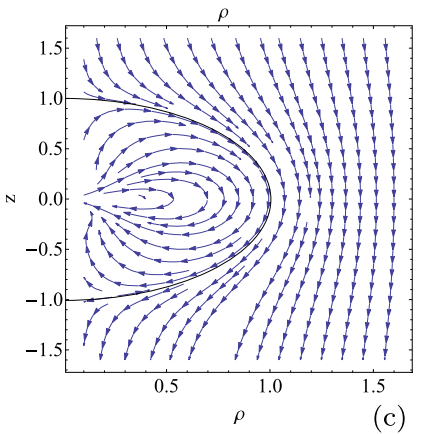

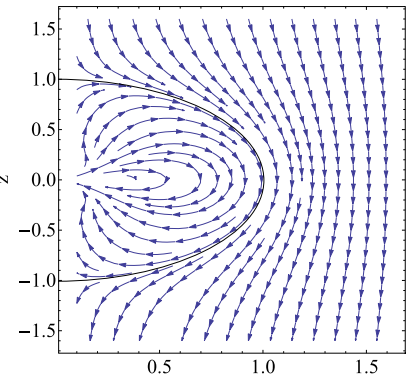

(b)

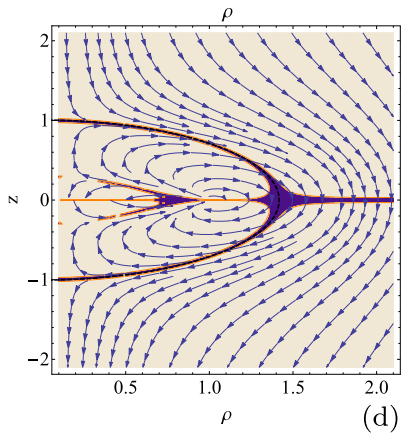

(d)
Figure 1. (a) The Wald solution in the cylindrical version of the BoyerLindquist coordinates for spin $a=0$. The event horizon is represented by the black semicircle. (b) The Wald solution in the Boyer-Lindquist coordinates for spin $a=0.99999$. (c) The Wald solution in the cylindrical version of the ingoing Kerr coordinates for spin $a=0.99999$; the coordinate transformations from Boyer-Lindquist do not affect the $(\rho, z)$ plane projection of the field lines. (d) The Wald solution in the Kerr-Schild coordinates for spin $a=0.99999$. The event horizon is still represented by a semicircle, but we have also shown an orange contour of the projection of the magnetic field onto the normal to the event horizon. The contour pieces straddle zero (the darker region corresponds to smaller projection values), so the flux density across the horizon actually vanishes, even though graphically it appears that the magnetic field lines thread through the horizon at places.

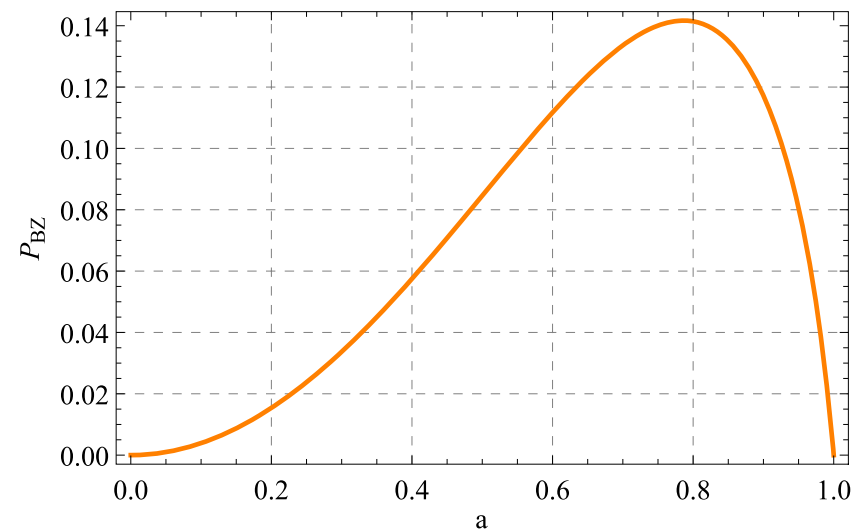

Figure 2. Power extracted from the black hole through the BZ process as predicted with Equation (1), setting black hole mass $M=1$ and the asymptotic magnetic field strength $B=1$.

redshift range of $0.4<z<2.1$ are close to 1 . Via a consideration of the quasar contribution to the X-ray background, Elvis et al. (2002) also arrived at the conclusion that "most supermassive black holes must be rapidly rotating."

The expulsion of the magnetic field by rapidly spinning black holes (named the black hole Meissner effect in analogy with superconductors) is therefore likely more than just a theoretical curiosity and has to be examined carefully if we want to understand real celestial objects. One would of course suspect that this effect is an esoteric feature only of the Wald solution. However, subsequent studies by Bicak \& Dvorak (1976), King (1977), Bicák \& Dvorák (1980), Bicak \& Janis (1985), Bicak \& Karas (1989), Karas \& Vokrouhlický (1991), Chamblin et al. (1998), Karas \& Budinova (2000), Bičák et al. (2007), Gibbons et al. (2013), Bičák \& Hejda (2015), and Hejda \& Bicak (2014) have greatly generalized the Wald solution to arbitrary axisymmetric solutions, to models containing the back-reaction of the electromagnetic field on spacetime, to higher dimensions, and so on, and this Meissner effect persists. A precise characterization of this effect is given by Bicak \& Janis (1985), that black holes in extremality (in spin, charge, or a combination of both) expel external axisymmetric, stationary magnetic fields. One may also ask the question that if the energy extraction process occurs in the ergosphere, would that render the Meissner effect irrelevant? Penna (2014) argued that the magnetic horizon flux density is required to ensure a clear passage for the negative-energy components in the Penrose-like BZ process to cross the horizon and thus remains a necessity. Pan \& Yu (2015) further showed that, when a perfectly conducting plasma is present, the magnetic field lines crossing into the ergosphere must also intersect with the horizon, so the Meissner effect would in fact expel the field lines all the way out of the ergosphere.

Despite the analytical predictions, numerical, general relativistic MHD simulations see no sign of this effect (McKinney \& Gammie 2004; McKinney et al. 2012; Tchekhovskoy et al. 2012; Contopoulos et al. 2013; Penna et al. 2013). Observationally, systems like microquasar GRS 1915+105 (spin 0.98) do not exhibit signs of jet quenching: their jet power is consistent with an $a^{2}$ scaling, showing no additional $a$-dependent suppression in magnetic flux density (Narayan \& McClintock 2012; Steiner et al. 2013). Therefore, mechanisms for evading the Meissner effect are needed, and several are already proposed. While examining arbitrary stationary solutions, Bicak \& Janis (1985) pointed out that solutions that are not axisymmetric do not experience the Meissner effect (briefly reviewed in Section 2 below). Later, Penna (2014) further identified the (split) monopole configuration as being able to evade it. ${ }^{1}$ Takamori et al. (2011) also found that currents carried by plasma in magnetospheres enable higher multipole magnetic fields to penetrate the horizon in the case of extremally charged (but nonspinning) black holes. On the other hand, if one allows an extremally spinning black hole to be charged, an induced magnetic dipole field can thread through the horizon (Bicák \& Dvorák 1980).

The remaining obvious loophole to exploit is the breaking of stationarity, which has escaped close scrutiny in previous studies concentrating on exact analytical solutions, due to the technical difficulty in handling fully dynamic electromagnetic fields in a curved spacetime. In Section 3 below, we utilize semianalytic arguments to show that intrinsic temporal variability (ITV) in the electromagnetic field can indeed serve as another route for the evasion of the Meissner effect. Furthermore, ITV may be a feature of prevalence among real

\footnotetext{
1 As is noted in Penna (2014), the infinite extremal Kerr throat argument utilized to elucidate the Meissner effect there is observer/coordinate dependent. We point out that Jacobson (2011) is a good reference that explains this issue. Also, the throat argument only requires the magnetic field lines to penetrate the horizon radially in the Boyer-Lindquist coordinates, saying nothing about their density's angular dependence. So while the monopole offers the simplest complying configuration, the results of Penna (2014) do not prohibit additional evasion mechanisms activating higher multipoles.
} 
astrophysical systems. From an observational perspective, we know that AGN and microquasar emissions are variable, so the electromagnetic fields surrounding astrophysical black holes are not stationary. From an energetics point of view, ITV brings about a more effective extraction of the black hole rotational energy, some of which would become available to fuel further dynamic behavior. So ITV may have a tendency to selfenhance (given the right conditions; see the end of Section 6 for more discussions) until an equilibrium with dissipation is reached (alternatively, if stable equilibrium is difficult to achieve, cycles of active and quiescent periods may alternate, causing ITV to appear intermittent). Together with the rotational energy, the angular momentum of the black hole is also extracted, so ITV can help prevent the astrophysical black holes from overspinning into naked singularities (or simply reaching extremality, which Aretakis $(2012,2013)$ and Lucietti \& Reall (2012) have suggested to be nonlinearly unstable), thus serving as an enforcer for cosmic censorship. In short, ITV may be an integral part of a self-consistent picture of astrophysical systems containing rapidly but subextremally spinning black holes.

Assuming this, it is then interesting to examine what kind of more detailed associations can be made between ITV and observations. One feature of ITV is that when it enhances the horizon magnetic flux, the additional energy extracted would go equitably into many sibling modes of differing angular quantum numbers (see Section 4). Thus an efficient "modecoupling" mechanism exists to promote the development of turbulence in the electromagnetic field, and subsequently ITV may be closely related to the stochastic microvariability of AGNs and microquasars. In Section 5, we discuss several potential applications to this observation.

1. (Section 5.1) ITV naturally produces a linear correlation between microvariability amplitudes and short-term mean fluxes, which has been recorded in numerous studies.

2. (Section 5.2) ITV possesses properties that make it useful in modeling the magnetic field structure observed in jets by polarization studies.

3. (Section 5.3) Using ITV and a photon leakage scheme, it is possible to obtain an improved theoretical fit to the X-ray microvariability power spectral density (PSD).

Finally, we conclude in Section 6. The derivations below are in geometrized units with $c=1=G$, unless otherwise specified.

\section{THE MEISSNER EFFECT}

We begin by reviewing the essential arguments of Bicak \& Janis (1985) that led to the observation of the Meissner effect being generic for any arbitrary, axisymmetric, stationary field configuration with $l \geqslant 1$. We will then adapt them to the case of temporally variable configurations in the next section and show that they also evade the Meissner effect, in much the same fashion as the rotationally asymmetric ones do. We will specialize to spin and not charge extremality, anticipating possible astrophysical applications. In addition, we will also restrict ourselves to vacuum (without plasma) solutions in the near-horizon regime, but allow arbitrary source charges and currents farther away, just as the original computation in Bicak \& Janis (1985) assumed. This is mostly to ensure that the calculations remain tractable. On the other hand, the derivation of the BZ process included the presence of a so-called forcefree plasma, in which the inertia of the charged particles is neglected because their stress-energy tensor is subdominant to that of the electromagnetic field. In reality, the particles do have mass and will not be able to travel at the speed of light and stay on the event horizon (a null surface by definition), so the particles sufficiently close to the horizon are on their way in, plausibly generating largely radial currents that do not greatly alter the radial component of the magnetic field and subsequently the horizon flux density, as compared to those in a very thin vacuum layer sourced by the same current and charge distribution outside (as the vacuum solutions examined below only need to satisfy horizon boundary conditions and we specialize to their limits when approaching the horizon, the vacuum layer can be very thin).

The arguments of Bicak \& Janis (1985; and Bicak \& Dvorak 1976 that they rely on) are based on the Newman-Penrose formalism. The underlying spacetime metric is that of Kerr:

$$
\begin{aligned}
d s^{2}= & -\frac{\Delta}{\Sigma}\left(d t-a \sin ^{2} \theta d \phi\right)^{2}+\frac{\Sigma}{\Delta} d r^{2}+\Sigma d \theta^{2} \\
& +\frac{\sin ^{2} \theta}{\Sigma}\left(\left(r^{2}+a^{2}\right) d \phi-a d t\right)^{2}, \\
\Delta= & r^{2}-2 M r+a^{2}=\left(r-r_{+}\right)\left(r-r_{-}\right), \\
\Sigma= & r^{2}+a^{2} \cos ^{2} \theta,
\end{aligned}
$$

where $\{t, r, \theta, \phi\}$ are the Boyer-Lindquist coordinates, $a$ is the dimensionless spin parameter, $M$ is the black hole mass, and $r_{+}$ is the radius of the event horizon. A particularly nice NewmanPenrose null tetrad (a basis to decompose vectors and tensors into their components) exists in this spacetime, namely the Kinnersley tetrad (Kinnersley 1969; see also, e.g., Zhang et al. 2012 for its properties), whose basis vectors are (expanded in the coordinate basis $\{d t, d r, d \theta, d \phi\})$

$$
\begin{aligned}
l_{a}= & \left(1,-\frac{\Sigma}{\Delta}, 0,-a \sin ^{2} \theta\right), \\
n_{a}= & \frac{1}{2 \Sigma}\left(\Delta, \Sigma, 0,-a \Delta \sin ^{2} \theta\right), \\
m_{a}= & \frac{1}{\sqrt{2} \Sigma}(a \sin \theta(a \cos \theta+i r), 0,-\Sigma(r-i a \cos \theta), \\
& \left.-\left(r^{2}+a^{2}\right) \sin \theta(a \cos \theta+i r)\right) .
\end{aligned}
$$

Under this tetrad, the Faraday tensor can be decomposed into three complex Newman-Penrose scalars $\Phi_{0}, \Phi_{1}$, and $\Phi_{2}$, representing the ingoing wave, Coulomb background, and outgoing wave pieces of the electromagnetic field, respectively, with the reconstruction formula being

$$
\begin{aligned}
F_{a b}= & 4 \Re \Phi_{1} n_{[a} l_{b]}+4 \Im \Phi_{1} m_{[a} \bar{m}_{b]} \\
& +4 \Re\left(\Phi_{2} l_{[a} m_{b]}\right)+4 \Re\left(\Phi_{0} \bar{m}_{[a} n_{b]}\right) .
\end{aligned}
$$

The magnetic flux across the horizon at $r=r_{+}$is simply the integral (Bicak \& Janis 1985)

$$
\left.\int_{\mathrm{EH}} F_{\theta \phi}\right|_{r=r_{+}} d \theta d \phi
$$

and the local flux density is thus essentially $\left.F_{\theta \phi}\right|_{r=r_{+}}$(aside from a $r_{+}^{2} \sin \theta$ factor from the integration measure). An examination of the Meissner effect is then a computation of $\left.F_{\theta \phi}\right|_{r=r_{+}}$(when we and the broader literature talk about vanishing flux in this context, it is really the flux density, not the total integral through the entire horizon, that is intended). 
Although we have used the Boyer-Lindquist coordinates for concreteness, we note that the tetrad is a geometrical construct that can be used in other coordinate systems as well. More importantly, $\left.F_{\theta \phi}\right|_{r=r_{+}}$is the same in Boyer-Lindquist as in Kerr coordinates (Bicak \& Dvorak 1976), so we are at liberty to compute it in the Boyer-Lindquist system, which is more convenient, but the result will nevertheless apply to horizonpenetrating coordinate systems.

In the extremal limit, $r_{-}$and $r_{+}$become identical, and $x=\Delta^{1 / 2}=r-r_{+}$becomes a measure of the distance to the horizon. To study whether the horizon flux density vanishes, one can then examine the leading order (lowest power) dependence of $\Phi_{0}, \Phi_{1}$, and $\Phi_{2}$ and the tetrad basis on $x$, and in turn that of $F_{\theta \phi}$ through Equation (4). The relevant tetrad scaling behavior can be read off directly from Equation (3), which are

$$
\begin{aligned}
& l_{\theta}=0, \quad l_{\phi} \sim x^{0}, \quad n_{\theta}=0, \quad n_{\phi} \sim x^{2}, \\
& m_{\theta} \sim x^{0}, \quad m_{\phi} \sim x^{0},
\end{aligned}
$$

so in order to achieve a nonvanishing horizon flux density, we need any of the three complex scalars to drop at or slower than

$$
\Phi_{0} \sim x^{-2}, \quad \Phi_{1} \sim x^{0}, \quad \Phi_{2} \sim x^{0} .
$$

Note that because of the singular behavior of the Kinnersley tetrad on the horizon, $\Phi_{0}$ is allowed to diverge without causing $F_{a b}$ to also become singular. On the other hand, the condition for $\Phi_{2}$ conflicts with the horizon regularity conditions (see, e.g., Brennan et al. 2013)

$$
\Phi_{0}: \mathcal{O}\left(x^{-2}\right), \quad \Phi_{1}: \mathcal{O}\left(x^{0}\right), \quad \Phi_{2}: \mathcal{O}\left(x^{2}\right),
$$

so it will not ever be satisfied; that is, no wave can come out of the black hole.

The generality of the Meissner effect as revealed by Bicak \& Janis (1985) boils down to the conclusion that the stationary axisymmetric solutions obey

$$
\Phi_{0} \sim x^{l-1}, \quad \Phi_{1} \sim x^{l}, \quad \Phi_{2} \sim x^{l+1} .
$$

So for $l \geqslant 1, F_{\theta \phi}$ vanishes on the horizon. To get this result, one solves the vacuum Teukolsky equation in the extremal and stationary limit, getting

$$
\Phi_{2}=\rho^{2} \sum_{l, m}{ }^{2} y_{l m} e^{i m \phi}-1 S_{l m}(\theta),
$$

with $\rho^{2}=M^{2} /(r-i a \cos \theta)^{2}$ and ${ }_{-1} S$ being the spinweighted spheroidal harmonics (which are simply the spinweighted spherical harmonics in the stationary case). The important term in Equation (10) is

$$
{ }^{2} y_{l m}=e^{-i m / x} x^{l+1} \xi\left(1-l,-2 l ; \frac{2 i m}{x}\right),
$$

where $m$ is the azimuthal quantum number and $\xi$ is the confluent hypergeometric function. ${ }^{2}$ After enforcing axisymmetry by setting $m=0$, both the exponential phase factor and $\xi$ in Equation (11) reduce to constants, and we recover the scaling in Equation (9) for $\Phi_{2}$. And to get to the other Newman-Penrose scalars from $\Phi_{2}$, derivatives against $x$ are

\footnotetext{
We note that $l=1$ corresponds to a pole of the confluent hypergeometric function $\xi$. Nevertheless, taking the limit of $l \rightarrow 1$ from other nearby values, we see that the solution remains valid in this case, so this result of Bicak \& Janis (1985) is valid for $l \geqslant 1$.
}

taken, at most once for $\Phi_{1}$, twice for $\Phi_{0}$, and thus the sequence of powers in Equation (9).

One immediately sees a way to evade the Meissner effect, namely through the breaking of axisymmetry. When $m \neq 0$ is allowed, the confluent hypergeometric function becomes a polynomial up to $1 / x^{l-1}$, so as far as the lowest power in $x$ is concerned, all of the different $l$ choices contribute at $x^{2}$ in Equation (11), rather than starting at higher powers of $x$ (thus more ignorable) for higher $l$ values, as in the $m=0$ case. This, however, is insufficient in itself for creating a nonvanishing flux at extremality, as $x^{2}$ is at the same order as the $l=1$ contribution when $m=0$, and we have seen that a dipole is not capable of creating nonvanishing fluxes in that situation. The key is the activation of the exponential phase term $e^{-i m / x}$. When we hit $e^{-i m / x} x^{p}$ with $\partial_{x}$, we get by the product rule a term $\propto\left(x^{p} / x^{2}\right) e^{-i m / x}$ when the derivative acts on the exponential. This gives a $x^{p-2}$ rather than a simple $x^{p-1}$, as a derivative on the polynomial part would otherwise produce, which makes it possible to create a scaling with sufficiently low power of $x$ to satisfy Equation (7). For example, with $l=3$, $m=3$, we have

$$
\begin{aligned}
{ }^{2} y_{33} & =e^{-3 i / x}\left(-\frac{36}{x^{2}}+\frac{60 i}{x}+30\right) x^{4}, \\
\frac{d^{2} y_{33}}{d x} & =6 e^{-3 i / x}\left(20 x^{3}+45 i x^{2}-42 x-18 i\right), \\
\frac{d^{2} y_{33}}{d x^{2}} & =\frac{18}{x^{2}} e^{-3 i / x}\left(20 x^{4}+50 i x^{3}-59 x^{2}-42 i x+18\right),
\end{aligned}
$$

so the first derivative, and subsequently $\Phi_{1}$, contains an $x^{0}$ order term, while the second derivative, and subsequently $\Phi_{0}$, contains an $x^{-2}$ order term, both capable of giving rise to a nonvanishing horizon flux density (see Equation (7)). This is technically why the Meissner effect only turns up in axisymmetry.

Finally, it is interesting to note that $l=0$ is not included in the derivation above and can serve as another way to evade the Meissner effect (Penna 2014). This corresponds to a magnetic monopole, which can be made more physical by placing a plasma-supported current sheet on the equatorial plane (so the magnetic field does not need to be continuous across it), splitting the spacetime into two halves with magnetic field configurations corresponding to oppositely charged monopoles on either side (the so-called split-monopole configuration of Blandford \& Znajek 1977). It is, however, worth noting that in the case of broken axisymmetry (and broken stationarity below), $F_{\theta \phi}$ from both $\Phi_{0}$ and $\Phi_{1}$ contributions can be nonvanishing on the horizon, while in the monopole case, only that from $\Phi_{1}$ is allowed to do so (the lowest multipole order for electromagnetic waves is dipole, so there is no ingoing monopolar wave). This means that if $\Phi_{0}$ as the ingoing wave is required for the energy extraction process (e.g., as the carrier of negative energy down the black hole), then the splitmonopole configuration may not suffice (note that although one of the original solutions in Blandford \& Znajek (1977) is called a split-monopole solution, because it is obtained by perturbing a monopole in Schwarzschild, the actual energy-extracting solution for slowly spinning Kerr that came out in the end has a nonvanishing $\Phi_{0}$ ). 


\section{EVASION THROUGH DYNAMIC EVOLUTION}

Dependence on the azimuthal coordinate $\phi$ is not the only way to produce an exponential phase term. In the Kerr spacetime, the temporal dimension has a fair amount of similarities with the azimuthal direction. For example, $\partial_{t}$ and $\partial_{\phi}$ are both Killing vectors of the underlying spacetime (the electromagnetic field does not need to possess all of the symmetries of the spacetime though, so it can break either axisymmetry or stationarity or both), and both $t$ and $\phi$ enter through simple exponential terms in the separation of variables expression for $\Phi_{2}^{3}$ :

$$
\Phi_{2}=\rho^{2} \sum_{l, m} \int d \omega e^{-i \omega t} e^{i m \phi}{ }_{-1} S_{l m \omega}(\theta)^{2} R_{l m \omega}(r) .
$$

Therefore, one is naturally led to the expectation that the breaking of stationarity may also result in an evasion of the Meissner effect.

The relevant equation now is the time-dependent version of the radial Teukolsky equation (Equation (4.9) of Teukolsky 1973):

$$
\begin{array}{r}
{\left[\frac{K^{2}+2 i(r-M) K}{\Delta}-4 i \omega r-\lambda_{\operatorname{lm} \omega}\right]} \\
+\Delta \frac{d^{2}\left({ }^{2} R_{l m \omega}\right)}{d r^{2}}=0
\end{array}
$$

with

$$
\begin{aligned}
K & =\left(r^{2}+a^{2}\right) \omega-a m, \\
\lambda_{\operatorname{lm} \omega} & =A_{l m \omega}+a^{2} \omega^{2}-2 a m \omega,
\end{aligned}
$$

where

$$
A_{l m \omega} \approx l(l+1)-\frac{a^{2} \omega^{2}}{2}\left(1-\frac{m^{2}}{l(l+1)}\right),
$$

when $a^{2} \omega^{2} / l(l+1)$ is small (see Equation (2.14) of Yang et al. 2013). We will make the small $|\omega|$ assumption below in order to obtain exact solutions, but we present numerical experiments and physical reasoning to argue that the important Meissner-effect-evading properties should be preserved for larger $|\omega|$ cases as well. Concentrating on a single constituent under the summation and integral signs of Equation (13), and dropping the subscripts for brevity, the extremal limit of Equation (14) for $m=0$ (we concentrate on evading the Meissner effect without breaking axisymmetry in this section)

\footnotetext{
3 As we are examining the effects of the fast oscillatory temporal variations here, and not the secular growth or decay that requires an accompanying energy change in the electromagnetic field, we concentrate on energetically steady evolutions with $\omega \in \mathbb{R}$. Nevertheless, we point out that when $\mathfrak{I} \omega \neq 0$ and if its value is oversimplified as compared to the real astrophysical situation, e.g., when we have a secular decay at a constant rate lasting for an unlimited duration, pathological behaviors such as a diverging amplitude in the infinite past may appear and may inject well-known unphysical pathologies into the horizon-limiting properties of $\Phi_{2}$ depending on the coordinate choice (see Jacobson (2011) and Section II C in Yang et al. (2015)). Furthermore, note that as we allow arbitrary currents and charges (including current sheets) outside the vacuum layer, we do not have the restrictive matched asymptotic expansion conditions seen for everywhere vacuum quasinormal modes that quantize $\omega$ (Teukolsky \& Press 1974).
}

is explicitly

$$
\begin{aligned}
& { }^{2} R(x)\left[x^{2}\left(-l(l+1)-\frac{\omega^{2}}{2}-4 i(x+1) \omega\right)\right. \\
& \left.+\left((x+1)^{2}+1\right) \omega\left(\left((x+1)^{2}+1\right) \omega+2 i x\right)\right] \\
& +x^{4}{ }^{2} R^{\prime \prime}(x)=0 .
\end{aligned}
$$

We immediately notice that the structure of Equation (17) is similar to that of the stationary and $m \neq 0$ case (e.g., it has a complex coefficient to ${ }^{2} R$ that is also present in the stationary $m \neq 0$ case, but not the $m=0$ case), which is

$$
x^{4}{ }^{2} R^{\prime \prime}(x)+{ }^{2} R(x)\left[-l(l+1) x^{2}+m(m-2 i x)\right]=0 .
$$

With Equation (18), a change of variable in the following form

$$
{ }^{2} R(x)=e^{-\frac{\zeta}{2}} \eta(\zeta)\left(\frac{D}{\zeta}\right)^{-l}, \quad \zeta=D / x,
$$

with the choice of $D=-2 i m$ turns it into a confluent hypergeometric equation

$$
-\zeta \eta^{\prime \prime}(\zeta)+(\zeta-2-2 l) \eta^{\prime}(\zeta)+\ln (\zeta)=0,
$$

whose solution appropriate for the near-horizon region is (Bicak \& Dvorak 1976)

$$
\eta=D^{2 l+1} e^{\zeta} \zeta^{-(2 l+1)} \xi(1-l,-2 l ;-\zeta),
$$

where the constant factor $D^{2 l+1}$ is introduced for convenience. With Equation (17), the same transformation (19) with the choice of $D=4 i \omega$ leads to

$$
\begin{aligned}
0= & \omega\left[-\zeta \eta^{\prime \prime}(\zeta)+(\zeta-2-2 l) \eta^{\prime}(\zeta)+\ln (\zeta)\right] \\
& +2 i \omega^{2} \eta(\zeta)-\frac{(15 \zeta+16) \omega^{3} \eta(\zeta)}{2 \zeta^{2}}-\frac{16 i \omega^{4} \eta(\zeta)}{\zeta^{2}} \\
& +\frac{16 \omega^{5} \eta(\zeta)}{\zeta^{3}}+\mathcal{O}\left(\omega^{6}\right),
\end{aligned}
$$

where we have expanded in the small quantity $\omega$. Keeping to the lowest order in $\omega$, we recover Equation (20). Therefore, the solution for the slowly varying time-dependent case is simply

$$
\begin{aligned}
\Phi_{2}= & \rho^{2} \sum_{l} \int d \omega e^{i \omega(2 / x-t)} x^{l+1}{ }_{-1} S_{l 0 \omega}(\theta) \\
& \times \xi\left(1-l,-2 l ; \frac{-4 i \omega}{x}\right),
\end{aligned}
$$

whose $x$-dependent terms are the same as those in Equation (11) with the simple replacement of $m \rightarrow-2 \omega$, so exactly the same mechanism for evading the Meissner effect applies. Specifically, the confluent hypergeometric function boosts the scaling contribution to $\Phi_{2}$ from all $l \geqslant 1$ multipoles from $x^{l+1}$ to $x^{2}$, and then the phase factor contributes additional $1 / x^{2}$ boosts once derivatives against $x$ are taken, allowing $\Phi_{1}$ and $\Phi_{0}$ to satisfy condition (7).

To probe the large $|\omega|$ behavior of Equation (17) (and to provide a visual example), we now turn to numerical solutions. We pick $l=4$ and $\omega=-2$ for demonstration, so that $a^{2} \omega^{2} / l(l+1)=1 / 5$ is still small and Equation (16) can be used but higher powers of $\omega$ itself are no longer negligible. The numerical solution to the full Equation (17) (without ignoring higher powers of $\omega$ ) with these parameters, together with analytical (from Equation (11)) stationary $m=0$ and $m \neq 0$ 

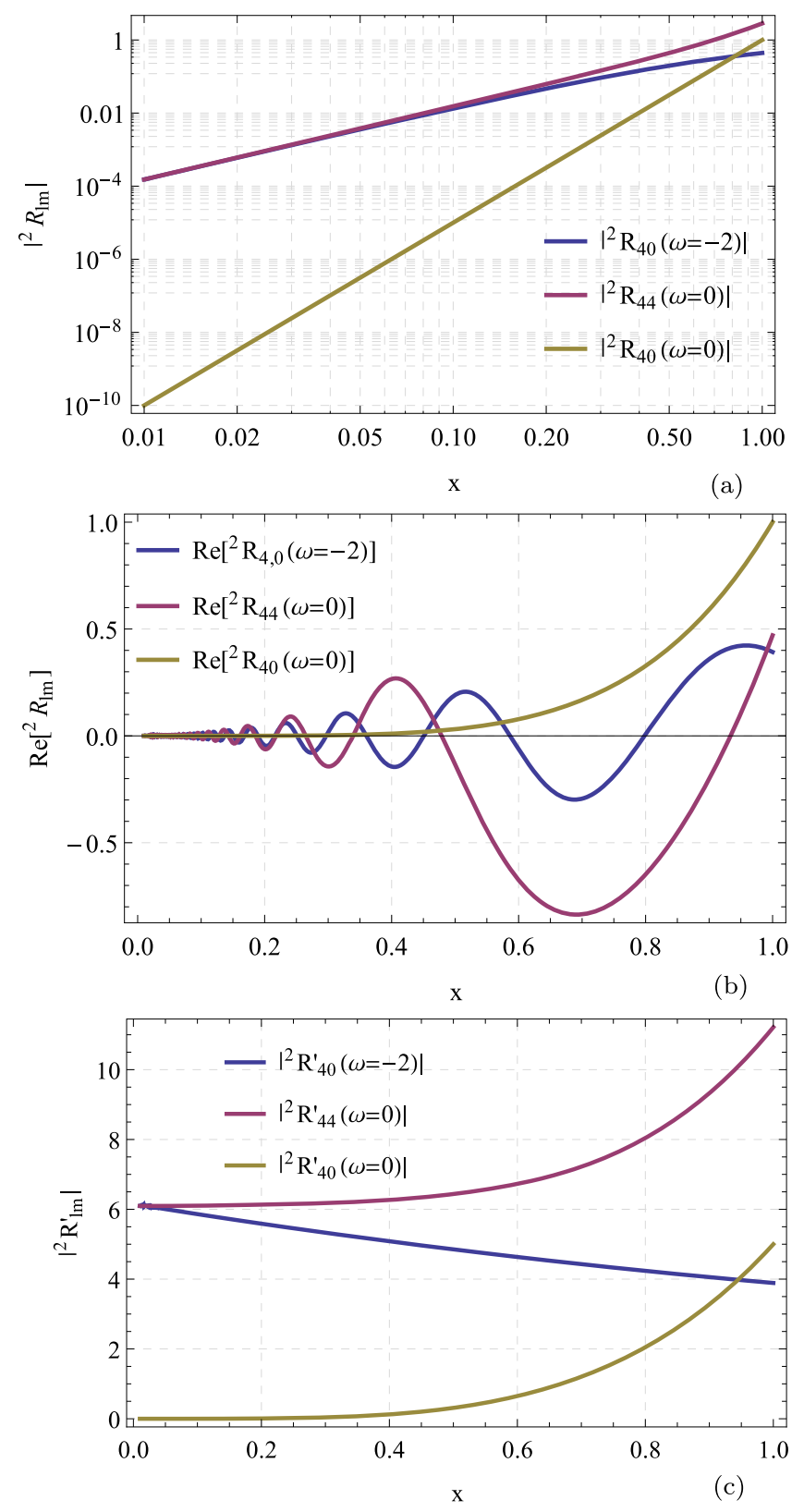

Figure 3. Radial component ${ }^{2} R$ of $\Phi_{2}$ for the cases with and without axisymmetry and stationarity (the solution to the time-dependent Equation (17) is obtained numerically). (a) In this $\log -\log$ plot, the slope of the lines corresponds to the power indices of power-law scalings. For $1<l=4$, the two stationary solutions do not share the same scaling behavior for $\left|{ }^{2} R\right|$. The axisymmetric case with $m=0$ scales as $x^{5}$, while the rotationally asymmetric case with $m=4$ has a scaling of $x^{2}$. The blue curve at small $x$ values coincides with, and is covered by, the purple one. Therefore the time-dependent $\left|{ }^{2} R\right|$ also scales as $x^{2}$. (b) Plotted are the $\mathfrak{R}\left({ }^{2} R\right)$ values. The breaking of either axisymmetry or stationarity produces oscillations with increasing frequency as we approach the horizon, symptomatic of a $1 / x$-dependent phase factor. (c) The first derivative of ${ }^{2} R$ shows that, just like the $m \neq 0$ case, the timedependent solution has a nonvanishing ${ }^{2} R^{\prime}$ in the horizon limit.

solutions as references, is plotted in Figure 3. To obtain the numerical solution, we need to specify ${ }^{2} R(x)$ and ${ }^{2} R^{\prime}(x)$ values at some $x=x_{0}$ as boundary conditions and then integrate Equation (17) away from there. The criteria for the admissibility of any particular boundary condition choice is that the resulting ${ }^{2} R$ should satisfy the horizon regularity condition (8), or in other words, $\left|{ }^{2} R\right|$ should scale as $\mathcal{O}\left(x^{2}\right)$. Looking at
Equation (22), we notice that the higher powers of $\omega$ tend to be accompanied by higher powers of $x$. So at very small $x$ values, one expects that even in the case of large $|\omega|$, the solutions to Equation (22) may be reasonably approximated by Equation (23). Therefore, we can try using Equation (23) to set the boundary conditions at some small $x_{0}$. We note that $x_{0}$ cannot be pushed all the way to the origin because Equation (22) is singular at $x=0$, due to the coefficient of the highest derivative term in it vanishing there. This also bleeds numerical issues into the exceedingly small $x$ regime $(x<0.01)$, making the acquisition of a reliable numerical solution difficult there (e.g., violent and unpredictable dependence on the working precision of the numerical routine is seen). Nevertheless, there is no problem with picking $x_{0}=0.01$ and then integrating away from the horizon. The solution thus obtained turns out to be reasonable, demonstrating a horizon-regularity-consistent $x^{2}$ scaling for $\left|{ }^{2} R\right|$ (Figure 3(a)). It also contains a phase oscillation with increasing frequency as we approach the horizon (Figure 3(b)). Furthermore, after a numerical derivative, ${ }^{2} R^{\prime}$ is explicitly seen to scale as $x^{0}$ (Figure $3(\mathrm{c})$ ), just as in the stationary but rotationally asymmetric case.

Our numerical experiment thus suggests that the important Meissner-effect-evading ingredients of ${ }^{2} R$ are not spoiled by large $|\omega|$. Aside from the technical observation that Equation (23) appears to provide reasonable, approximate large $|\omega|$ solutions in the important small $x$ regime (so a better-but not rigorously proven-characterization for Equation (23) is perhaps that it is valid when either $|\omega|$ or $x$ is small), we can also make physical arguments as to why it should be so. For example, the $x^{2}$ scaling for $\left|{ }^{2} R\right|$ even when $l>1$ is not surprising because it is the minimal requirement for the horizon regularity condition (8) to be satisfied, so it is the least special or restricting and should be generic among field configurations that are not severely constrained by, for example, multiple symmetries. Equivalently stated, further restricting the field to satisfy (8) beyond the minimal order is unnecessary and wasteful as far as horizon regularity is concerned, so such restrictions, if they exist, have to come from other considerations. More importantly, the physical meaning of the oscillatory phase factor in the time-dependent case is even more apparent than in the rotationally asymmetric case. This factor simply enforces an ingoing-wave boundary condition at the event horizon as demanded by causality:

$$
{ }^{2} R \propto e^{-i \omega\left(t+r_{*} / v\right)},
$$

where $v>0$ is the radial velocity, and $r_{*}$ is the tortoise coordinate that can be seen as a function of $x$ defined by $d r_{*} / d x=\left(r^{2}+a^{2}\right) / \Delta$, which is approximately $2 / x^{2}$ near the horizon, and so $r_{*} \sim-2 / x$ with an appropriate integration constant. Specifically for a rotating black hole, ${ }^{2} R$ asymptotes to (see, e.g., Yang et al. 2013)

$$
{ }^{2} R \propto e^{-i\left(\omega-m \Omega_{H}\right) r_{*}} e^{-i \omega t},
$$

on the horizon, where $\Omega_{H}$ is the horizon angular velocity. Setting $m=0$, Equation (25) gives us the phase factor in Equation (23). In addition, because the ingoing-wave condition (25) is generic for all solutions of the original unsimplified radial Teukolsky equation, the same factor should also appear in ${ }^{2} R$ even when $|\omega|$ is large. On the other hand, setting $\omega=0$ and noting that $\Omega_{H}=1 / 2$ at extremality, Equation (25) reduces to $e^{-i m / x}$, agreeing with Equation (11) for the 
stationary and rotationally asymmetric case discussed in Section 2.

\section{MODE COUPLING VIA ENERGY INJECTION}

Within our discussion, force-free currents, which are nonlinear functions of the electric and magnetic fields, should be present outside the boundary layer and have the potential to bring about coupling between time-dependent perturbations characterized by different angular quantum numbers $l$ (abbreviated to "modes" below) and induce turbulence when given the right conditions. Analytical studies (Goldreich \& Sridhar 1995) and numerical simulations (Cho 2005; Zrake \& East 2015) in the flat spacetime show that force-free turbulence possesses a Kolmogorov energy spectrum with power index $-5 / 3$. However, for freely decaying MHD (not magnetically dominated) turbulences, Zrake (2014) and Brandenburg et al. (2015) have measured indices closer to -2 .

Aside from such direct nonlinear couplings, there may be indirect energy-injection effects that are also capable of "coupling" the modes, in the sense that a single seeding mode can excite siblings. Essentially, the spherical harmonic content of the electromagnetic perturbation appearing in the NewmanPenrose scalars are not faithfully reflected in the horizon flux density because, in order to reconstruct the Faraday tensor according to Equation (4), one has to involve the NewmanPenrose basis vectors, which are themselves functions of $\theta$. For concreteness and some variety in coordinate choice, let us consider ingoing Kerr coordinates $(\tilde{t}, r, \theta, \tilde{\phi})$, which share the same $r$ and $\theta$ with the Boyer-Lindquist coordinate system, while the relationship for the temporal and azimuthal coordinates are

$$
\tilde{t}=t+r_{*}-r, \quad \tilde{\phi}=\phi+\frac{a}{r_{+}-r_{-}} \ln \left|\frac{r-r_{+}}{r-r_{-}}\right| .
$$

The metric is now given by

$$
\begin{aligned}
d s^{2}= & -\left(1-\frac{2 M r}{\Sigma}\right) d \tilde{t}^{2}+H^{2} d r^{2}+\Sigma d \theta^{2} \\
& +\frac{A \sin ^{2} \theta}{\Sigma} d \tilde{\phi}^{2}-2 a H \sin ^{2} \theta d r d \tilde{\phi} \\
& -\frac{4 a M r \sin ^{2} \theta}{\Sigma} d \tilde{t} d \tilde{\phi}+\frac{4 M r}{\Sigma} d r d \tilde{t}
\end{aligned}
$$

where

$$
\begin{aligned}
& A=\left(r^{2}+a^{2}\right)^{2}-a^{2} \Delta \sin ^{2} \theta, \\
& H=\sqrt{1+\frac{2 M r}{\Sigma}} .
\end{aligned}
$$

Using Equation (26), it is straightforward to compute the Jacobian for the coordinate transformation and subsequently the expressions for the Kinnersley tetrad in the ingoing Kerr coordinates; then using Equation (4), we obtain that for a, e.g., $\Phi_{0}$ perturbation to the Wald solution, the horizon flux density is given by (setting $M=1$ for brevity)

$$
F_{\theta \tilde{\phi}}=2 \sqrt{2} \Re\left(\frac{((r-2) r+16) \sin ^{2} \theta}{r-4 i \cos \theta} \Phi_{0}\right),
$$

where the extra $\theta$-dependent term multiplying onto $\Phi_{0}$ makes it clear that even when $\Phi_{0}$ contains a single solitary $l$ contribution, the horizon flux density will in general contain both higher and lower $l$ values.

Fundamentally, both the Newman-Penrose scalars and $F_{\theta \tilde{\phi}}$ are components of the Faraday tensor, but under different tetrads, with the former being associated with the Kinnersley tetrad and the latter under the coordinate tetrad $\{d \tilde{t}, d r, d \theta, d \tilde{\phi}\}$. The $\theta$-dependent transformation between the tetrads then causes a mismatch between the harmonic decompositions of the tensor components in these respective bases. This mismatch has physical consequences. Because the Maxwell equations are separable only in the Kinnersley tetrad, it is the $l$ of the Newman-Penrose scalars that should be used to label the electromagnetic evolution modes. On the other hand, $P_{\mathrm{BZ}}$ is proportional to flux squared, so it supplies energy according to the harmonic components in $F_{\theta \tilde{\phi}}$. There is no reason to expect this alteration in harmonic contents to get exactly reversed when the extracted energy is fed back into the electromagnetic variation modes, that is, for the energy to simply proportionally enhance the original seeding mode. Although a rigorous analytical proof for this energy-extraction detail is not currently available ${ }^{4}$, we note that for the extracted energy to leave the horizon, outgoing $\Phi_{2}$ must be involved even when the seed field is purely ingoing containing only $\Phi_{0}$, so the field configuration has to change more than just an overall amplitude. Therefore, energy most likely gets pumped into other sibling modes that may not be present initially. This sequence then repeats, and energy cascades and inversecascades into a broad range of available length scales, eventually establishing a steady turbulent spectrum.

For a more visual demonstration, consider an ingoing perturbation in $\Phi_{0}$ of the simple toy form

$$
\Phi_{0}=\frac{1}{2} \frac{e^{-i \omega(\tilde{t}+r)}}{\left(r-r_{+}\right)^{2}}{ }_{1} Y^{l, m=0}(\theta, \tilde{\phi}),
$$

on top of a background Wald solution, where ${ }_{1} Y^{l, m}$ are the spinweighted spherical harmonics (we choose $\omega=0.01$ so $a \omega \ll 1$, and the spherical harmonics are good approximates to the spheroidal harmonics, for which we do not have simple analytical expressions). Note that Equation (30) satisfies the ingoing boundary condition (25) (noting $\tilde{t}+r=t+r_{*}$ ), but does not properly solve the radial Teukolsky equation everywhere (we do not have exact solutions for $\Phi_{0}$ in the dynamic case). In any case, though, it is the angular behavior that interests us in this section, so the radial part just needs to be sufficiently large near the horizon. We then use Equation (4) to reconstruct the Faraday tensor and plot the perturbed magnetic field lines in Figure 4. We can also estimate the spherical harmonic components in the horizon flux density, which is plotted in Figure 5 and shows that higher $l$ values are generically present even when the initial perturbation consists of only $l=1$. In this case, there are not many modes with even lower $l$, so the "leaking" is asymmetric. In contrast, high initial $l$ perturbations generate both lower and higher $l$ components in the flux density fairly symmetrically (although the lower $l$ side

\footnotetext{
4 Even the stationary monopole-like BZ solution for rapidly spinning black holes is still not known analytically, although its power should remain proportional to magnetic flux density squared on dimensionality grounds because even though correction terms with higher powers of $a$ may appear, $a$ is itself dimensionless, so we still need magnetic field squared to create an energy-density-like coefficient.
} 


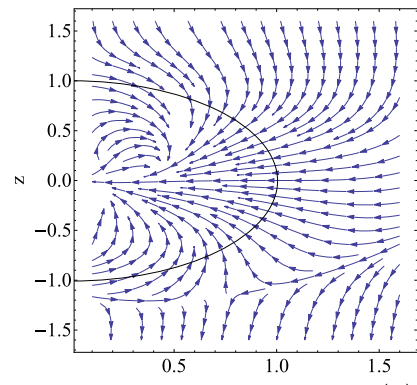

$\rho$

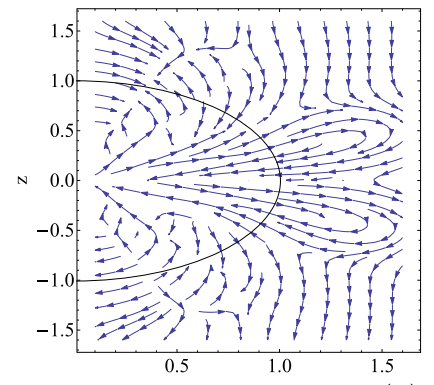

(b)
Figure 4. Perturbed magnetic field lines in the cylindrical counterpart to the ingoing Kerr coordinates, and with $\tilde{t}=0, \omega=0.01$, and $a=0.99999$ : (a) Adding an $(l=1, m=0)$ mode $\Phi_{0}$ to the Wald solution introduces a change in the magnetic field line directions, which now thread through the horizon while originally flowing around it as in Figure 1(c). (b) Higher $l=10$ perturbing mode in $\Phi_{0}$ also causes the field lines to thread through the horizon. However, more lines become closed, emerging and returning to the horizon, rather than extending out to infinity.

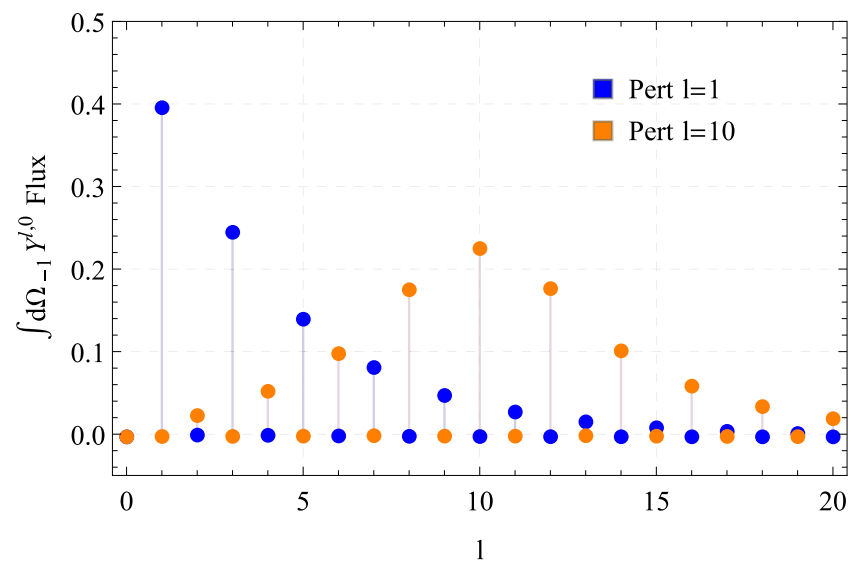

Figure 5. Inner product of the flux density across the horizon (resulting from an $l=1$ or $l=10$ seed perturbation in $\Phi_{0}$, as depicted in Figure 4) with spinweighted spherical harmonics ${ }_{-1} Y^{l, 0}$ (appropriate for $\Phi_{2}$ that carries energy outward). A significant presence of higher $l$ modes is seen in the flux even when the initial perturbation contains only $l=1$. With initial perturbation of a higher $l=10$, harmonic contributions leak into both higher and lower $l$ values in a more symmetric fashion. The inner product values are normalized, so contributions from all of the modes for, say, the $l=1$ seed, add up to unity.

is still cut off at $l=0$ ). As it is reasonable to expect more patchy energy patterns from higher $l$ components in $F_{\theta \tilde{\phi}}$ to be better matched and thus absorbed by corresponding higher $l$ modes of the electromagnetic field, this flux density harmonic decomposition pattern can be seen as an approximate surrogate for the mode excitation pattern, and the overall trend is a spreading of energy among different $l$ values.

Although this energy-injection effect looks fairly different from the usual direct mode-couplings (if we view our modes as driven oscillators, then they are coupled through the driving force's dependence on other modes), it nevertheless represents a robust and efficient mechanism for cascading and inversecascading energy throughout the entire spectrum, exciting previously absent modes to establish rich angular structures in

\footnotetext{
5 We expect the emergence of a great many current sheets (where electric field accelerates charges, leading to a transfer of the electromagnetic field energy into thermal energy) when the magnetic field lines become highly fragmented, as large curl in $\boldsymbol{B}$ encourages growth in $\boldsymbol{E}$. This expectation appears to be verified by Zrake \& East (2015), who saw a reduction in the rate of dissipative losses when energy is transferred to larger length scales.
}

the electromagnetic field that evolve with even richer dynamics. On the other hand, we also have dissipative processes that are likely more prominent in a high $l$ environment. ${ }^{5}$ We do not know of the quantitative details of the energy injection and dissipation processes, or how large the contributions from other coupling mechanisms are in comparison, but if we assume that in a steady turbulent state (in which the aforementioned energy gains and losses are balanced), the energy spectrum is only a function of the variation frequency $\omega$ and the rate of energy dissipation per unit volume, then one can argue for a Kolmogorov $-5 / 3$ power-law dependence on $\omega$ on dimensional grounds. In reality, the situation may be more complex, with the power index not being exactly $-5 / 3$, or the steady state being only quasi-steady, exhibiting boom-and-bust cycles when the energy injection or dissipation process temporarily overpowers the other. Sophisticated numerical simulations are needed to produce a more accurate characterization.

\section{OBSERVATIONAL IMPLICATIONS}

There are several alternatives for evading the black hole Meissner effect associated with extremal spin: the (split) monopole configuration (if its lack of $\Phi_{0}$ poses no problem), the breaking of axisymmetry, having the black hole carry electric charge, ITV, and possibly others. We will discuss some potential observational consequences of ITV, but note that other evasion mechanisms would likely be present simultaneously. Of those, the monopole configuration may be the more common because astrophysical black holes are expected to be electrically neutral, and there is no obvious reason to expect their spin axes to be systematically misaligned with the magnetic field (Penna 2014). We further note that although monopoles are not natural for jet formation, gradual collimation occurring farther away from the black hole horizon can possibly compensate (Junor et al. 1999).

On the other hand, as mentioned in Section 1, we expect ITV to be rather ubiquitous among systems powered by rapidly spinning holes. In what follows, we will restrict our discussion to AGNs of this character (amounting to most of them, according to the estimates of Reynolds (2013), Wang et al. (2006), and Elvis et al. (2002)). Furthermore, black hole X-ray binaries such as Cygnus $\mathrm{X}-1$ are assumed to have much the same energy generation and emission processes as AGNs and are as such named microquasars. Our discussion below will apply to microquasars the same way it does to AGNs, and we will utilize either class of objects depending on available observational data.

There are a plethora of different AGN categories defined according to their observational signatures, but schemes have been devised to combine them into a single unified picture. One leading contender is based on the orientation of the jets (Bregman 1990; Antonucci 1993; Urry \& Padovani 1995). Specifically, most or all AGNs contain jets, an accretion disk, and an axisymmetric dusty torus. The disk material heats up and radiates thermally in the optical to ultraviolet bands. Furthermore, a corona of hot material above the disk can inverse-Compton scatter softer photons up to X-ray energies. The jet also radiates, from radio to gamma-ray bands through synchrotron and inverse-Compton processes, so the radiations are nonthermal. When a jet is pointed close to our line of sight (as in the case of blazars), we can see much nonthermal radiation produced inside the jet, in addition to that originating 
from the disk and corona. For example, X-ray continuum emissions from the jet and the corona share a common scattering origin, while radio signals are considered to be from the jet alone. On the other hand, if the jet is pointed elsewhere, thermal radiations from the disk become more prominent, and we also need to be careful about scattering by the dusty torus.

A particularly interesting aspect of AGNs and microquasars is that they exhibit luminosity variations. Despite the current poor understanding of the underlying mechanism for this phenomena (Wold et al. 2007), it is considered to be of great importance as it may help diagnose the fundamental physics behind the various emission activities (Hook et al. 1994). Of the many constituents of the central core of AGNs and microquasars, previous explanations for variability have largely concentrated on the disk in order to take advantage of its rich dynamics. Black holes, on the other hand, are rather unlikely to spontaneously vary because radiating away its time dependence through quasinormal ringing appears to be the norm. There is, however, another constituent that has been subjected to less scrutiny: the electromagnetic field threading the black holes, which is quite capable of having its own intrinsic nontrivial dynamic evolution in a curved spacetime (the dynamics can initially be triggered by other processes though). In other words, variabilities driven by ITV are an enticing possibility.

An ITV-based explanation would corroborate with the assertion that variability is an important tool for probing AGN and microquasar energetics because ITV ties in to the very core of the black hole rotational energy extraction process and directly regulates the extraction efficiency, which also means it is capable of generating the type of large variation amplitudes seen observationally. In addition, features of the electromagnetic field naturally travel at the speed of light and are thus capable of creating the extremely short timescales that are on the order of light-crossing times. Indeed, it is due to the prevalence of such short timescales (not only in the variations themselves, but also in lags between different wavebands, and so on) that Gaskell (2004) speculated that the mechanism underlying variations should be electromagnetic in nature (although it is electromagnetic processes in the disk, rather than in the vicinity of the black hole, that are envisioned there). In the next three sections, we propose several possible links between ITV and observations in the context of variability and other related issues.

\subsection{Variability-Flux Correlation}

Perhaps the most straightforward prediction of ITV is that, because it is invoked to increase the energy-extraction efficiency of the BZ process, one can expect AGNs and microquasars to become more luminous during those periods of pronounced variation. Furthermore, as ITV occurs in the core region in the vicinity of the black holes, the relevant timescale is the light-crossing time across a Schwarzschild radius (around $1 \mathrm{hr}$ for a $10^{8} M_{\odot}$ supermassive black hole), so we expect that the microvariability ${ }^{6}$ is the most relevant for our discussion.

Uttley \& McHardy (2001), Uttley et al. (2005), Superina \& Degrange (2008), Dhalla et al. (2010), Vaughan et al. (2003),

\footnotetext{
6 Variability categories are long term (on a timescale of several years), short term (months to weeks), and micro (hours to minutes; see, e.g., Gupta et al 2008). One may also distinguish between the longer term and microvariabilities according to whether the bluer-when-brighter feature is seen in the variations (Sasada et al. 2008), in which case microvariability also includes shorter-period multiday cases. It is this latter, more physical definition that we will adopt here
}

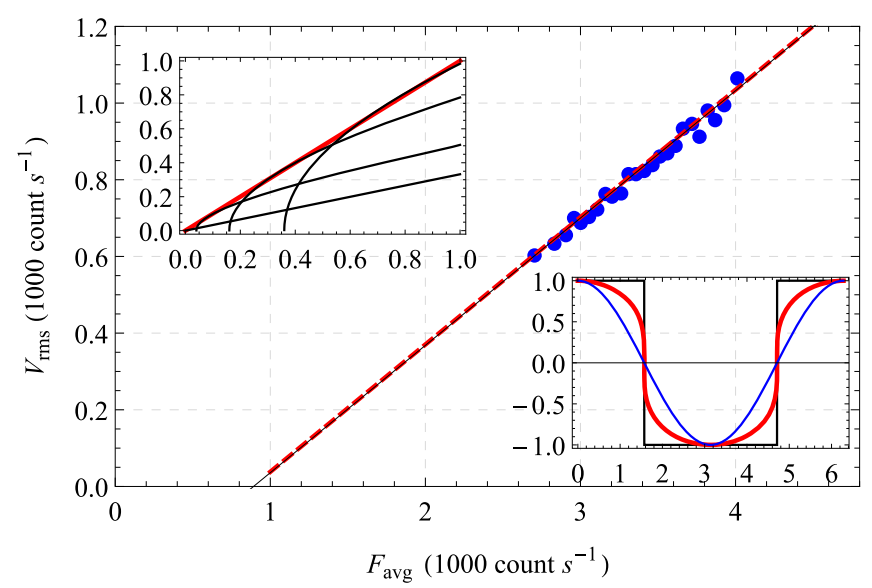

Figure 6. Mean flux vs. root mean square variation plot for the Cygnus X-1 microvariability. The blue dots are the observational data, which are taken from Figure 1 of Uttley \& McHardy (2001). The dashed red line is the fitting result using Equation (35), and the thin black line (beneath the red line) represents the best linear fit. The top-left inset displays curves derived from Equation (35) with $F_{c}=0$ and $\mathcal{F}_{m} \neq 0$. Those associated with larger $\mathcal{F}_{m}$ values are farther to the top-right. The bottom-right inset depicts the magnetic flux density variation profile $f(\cos (t))$ as given by $p$ value choices of 0 (black), 0.28 (red, thick, and the same value used for the red line in the main figure), and 1 (blue). The horizontal axis for the bottom-right inset is time.

and Gaskell (2004) indeed observed a positive correlation between the root mean square amplitude of the microvariations and the average flux during the variation periods (not the longterm secular brightness, which would be a different study unrelated to the present discussion) for Seyfert galaxies and microquasars in the X-ray band. In particular, Uttley \& McHardy (2001) recorded a strikingly clean positive linear correlation for Cygnus X-1 (spin $>0.95$; see Figure 1 therein, reproduced in Figure 6 below), with a positive intercept on the mean flux axis, corresponding to a variability-independent component amounting to about a quarter of the maximum flux value.

For other scenarios, similar linear correlations are also recorded in blazars by Giebels \& Degrange (2009) and Gaur et al. (2010), again in the X-ray band. However, in this case, the data points are much more scattered (due to the inherent difficulty in detecting such relations in blazars because they are faint with large Poisson noise and sampled intensely only during flaring events (Giebels \& Degrange 2009)), and we do not know if the correlation is still linear.

In the optical bands, Lyutyj \& Oknyanskij (1987) observed a linear correlation for Seyfert galaxy NGC 4151, which is also very clean (see Figure 5 therein) and with a positive intercept on the flux axis. For blazar W2R1926+42, Edelson et al. (2013) reports a very strong linear relationship (Figure 6 therein), and once again a positive flux axis intercept is seen. In the gamma-ray emissions, Degrange et al. (2008) also observed a linear correlation for the microvariability of blazar PKS 2155 304 (Figures 7 and 8 therein).

For the sake of completeness, we note that another way to examine whether there is a positive correlation between fluxes and variation amplitudes is through the shape of the distribution for the latter. Modeling the flux $F$ exhibiting microvariations as a stochastic process,

$$
\frac{1}{F^{p}} d F=\mu d t+\sigma d W
$$


where $\mu$ is a long-term drift, $\sigma$ is the short-term volatility, and $W$ is the Wiener process; then a positive correlation corresponds to $p>0$. In particular, if $p=1$ (linear correlation), the distribution for $F$ will be lognormal (see Figure 5 in Edelson et al. 2013), while for more general $p>0$, we will have a distribution skewed toward higher $F$, as signified by a positive skewness (third cumulant over the $3 / 2$ power of the second cumulant). This method would produce more apparent results when the variance $v$ of the data is large. For example, with a lognormal distribution, the skewness is

$$
\sqrt{e^{v}-1}\left(2+e^{v}\right)
$$

This means that it may be difficult to obtain reliable results through this type of integral measurements when fluctuation amplitudes are small (a possible contributing factor to the inconclusive or negative results in some studies looking for lognormal distributions, e.g., Mocanu \& Sándor 2012), in which case an alternative measurement through binning data and plotting the standard deviation versus mean in each bin would likely be more effective and is indeed adopted by most studies mentioned above.

We show now that the ITV-induced variability naturally produces such linear-looking correlations. Recall that the power from the BZ process is proportional to the magnetic horizon flux density squared (see, e.g., Equation (1) and footnote 4), and assume that the energy flux reaching Earth is ultimately traced back to a region on the horizon, whose average flux density is approximated by the illustrative toy model (we will generalize later):

$$
\mathcal{F}(t)=\mathcal{F}_{m}+\mathcal{F}_{t} f(\cos (\omega t)),
$$

where $\mathcal{F}_{m}$ is the monopole contribution, and $\mathcal{F}_{t}$ is the magnitude of the ITV contribution that we can vary to represent different microvariability amplitudes (we can also add in more ITV components with different frequencies, which will not change the qualitative features below). The function $f$ provides flexibility to the profile of temporal variation (as it is not necessarily sinusoidal). Specifically, we assign it the simple form

$$
f(x)=\frac{x}{|x|}|x|^{p}
$$

so when $p=0, \mathcal{F}(t)$ jumps between two discrete values, while larger $p$ provides smoother transitions. Now the average flux (over one or more oscillation periods) and root mean square (rms) variation (not rms of flux; we take away the mean from the flux before the rms computation to match Uttley \& McHardy 2001) are given by

$$
\begin{aligned}
F_{\mathrm{avg}}= & \mathcal{C}\left(\mathcal{F}_{m}^{2}+\mathcal{F}_{t}^{2} \frac{\Gamma(p+1 / 2)}{\sqrt{\pi} \Gamma(p+1)}\right)+F_{c}, \\
V_{\mathrm{rms}}= & \frac{\mathcal{C} \mathcal{F}_{t}}{\sqrt{\pi}}\left[\mathcal{F}_{t}^{2}\left(\frac{\sqrt{\pi} \Gamma(2 p+1 / 2)}{\Gamma(2 p+1)}-\frac{\Gamma(p+1 / 2)^{2}}{\Gamma(p+1)^{2}}\right)\right. \\
& \left.+\mathcal{F}_{m}^{2} \frac{4 \sqrt{\pi} \Gamma(p+1 / 2)}{\Gamma(p+1)}\right]^{1 / 2},
\end{aligned}
$$

where $\mathcal{C}$ is a constant depending on the black hole mass and spin as well as the latitude $(\theta)$ of the region under consideration, $\Gamma$ is the Euler gamma function, and $F_{c}$ represents a steady (time-independent) flux contribution from non-BZ processes, such as the disk-driven one in Blandford \& Payne (1982).

Equation (35) possesses the following properties that are used to produce the fitting to observational data in Figure 6.

1. The relationship between $F_{\text {avg }}$ and $V_{\text {rms }}$ is approximately linear (regardless of $p$ choices) when $\mathcal{F}_{t}$ is large as compared to $\mathcal{F}_{m}$, but not so when $\mathcal{F}_{t}$ is small, so an adjustment to the linear intercept can be expected (see the top-left inset in Figure 6, where curves farther to the topright correspond to larger $\mathcal{F}_{m}$ and show greater adjustments).

2. The external $F_{c}$ simply shifts the entire $V_{\text {rms }}$ versus $F_{\text {avg }}$ curve toward the larger $F_{\text {avg }}$ side. On the other hand, the monopole contribution $\mathcal{F}_{m}$ not only shifts the curve horizontally, but also vertically, so the curve always touches the $V_{\mathrm{rms}}=F_{\text {avg }}$ bisection line (see the top-left inset in Figure 6). Subsequently, using $\mathcal{F}_{m}$ alone, we cannot give the linear approximation to the curve a positive intercept on the $F_{\text {avg }}$ axis, as is demanded by the data. Therefore, during fitting, we have used only $F_{c}$ as a variable and simply set $\mathcal{F}_{m}=0$, while noting that future observational data in the very low $F_{\text {avg }}$ regime are needed to ascertain whether $\mathcal{F}_{m}$ is in fact present.

3. The power $p$ in Equation (34) determines the slope of the $V_{\text {rms }}$ versus $F_{\text {avg }}$ curve at large $\mathcal{F}_{t}$, with $p=0$ corresponding to a vanishing slope, and $p=1$ to a slope of $1 / \sqrt{2}$. For the observational data in Figure $6, p=0.28$ provides the best fit. The $f(\cos (t))$ with aforementioned choices of $p$ are shown in the bottom-right inset of Figure 6.

4. There is a degeneracy between $\mathcal{C}$ and $\mathcal{F}_{t}$, so we simply set $\mathcal{C}=1$ and use $\mathcal{F}_{t}$ alone for the fitting. The result is the red dashed line in Figure 6, which is indistinguishable from the best linear fit (solid black line).

Note that although we have used an oversimplified, deterministic temporal evolution profile for $\mathcal{F}(t)$ for demonstration, the appearance of linear correlation does not depend on this choice. The essence is that the same process for generating the variation is responsible for creating (a part of - we will ignore $\mathcal{F}_{m}$ and $F_{c}$ below for brevity) the mean flux, which is always true with ITV, so both are proportional to the same amplitude parameter, which is $\mathcal{F}_{t}^{2}$ in our case. For example, if we let $\mathcal{F}(t)=\mathcal{F}_{t} g(t)$ with $g(t)^{2}$ be some fundamental stochastic process in time (more appropriate for a turbulent ITV) so it has fixed mean and variance for each-and across_-data binning periods of equal duration (different periods can of course differ in their "volatility" $\mathcal{F}_{t}$ ) and let $\langle\cdot\rangle$ represent averaging over a binning period, then we have

$$
\begin{aligned}
F_{\mathrm{avg}} & =\mathcal{C} \mathcal{F}_{t}^{2}\left\langle g(t)^{2}\right\rangle, \\
V_{\mathrm{rms}} & =\mathcal{C} \mathcal{F}_{t}^{2} \sqrt{\left\langle\left[g(t)^{2}-\left\langle g(t)^{2}\right\rangle\right]^{2}\right\rangle} .
\end{aligned}
$$

So clearly we still have a linear relationship between $F_{\text {avg }}$ and $V_{\mathrm{rms}}$, with the slope determined by the cumulants of $g(t)^{2}$ (their counterparts in the deterministic case earlier are adjustable with $p$ ). 


\subsection{Manifestation in Jet Emission}

The jets of AGNs and microquasars are complicated objects involving much as-yet-unknown physics (Hardee 2008), but their polarized nonthermal radiation allows for examination of the magnetic field structure within (Begelman et al. 1984; Wardle 2013), to which ITV in the electromagnetic field may be relevant. Indeed, the magnetic field structure of the quiescent (without the shocks of Blandford \& Königl (1979) that are often invoked to explain long-term variabilities) jet flow appears to be highly nontrivial. On the one hand, an ordered magnetic field may be required for maintaining the jets' structural integrity (see e.g., Rosen et al. 1999). On the other hand, observations such as those on the inhomogeneous linear polarization in radio signals (Jones 1988) are consistent with there being many randomly oriented magnetic cells (Hughes 2005; Hughes et al. 2011). Therefore, one expects both ordered and random components of the magnetic field to be present.

The orderliness and randomness are intertwined, but a general trend can be gleaned from observational data. The existence of an ordered toroidal component to the magnetic field is supported by rotation-measure gradient records (Wardle 2013). A possibility is that a largely toroidal field surrounds the jet, stabilizing it against disruptive Kelvin-Helmholtz instabilities through magnetic tension. Such a toroidal field can be produced by currents flowing along the jet and the backflow (Figure 14 of Begelman et al. 1984). In the jet spine (middle in the transverse direction), some polarization measurements have demonstrated that the dominant magnetic field direction is along the jet (Wardle 2013), although it is unlikely to be unidirectional (Begelman et al. 1984). A possibility is then that inside the toroidal field and in the jet spine, we have sheared magnetic loops produced by a plasma velocity gradient transverse to the jet direction, stretching loops frozen in the plasma into elongated shapes along the jet, thus enhancing the poloidal fields (see Figures 12 and 13 of Begelman et al. (1984) for a visual depiction). The magnetic field in the spine is otherwise random, as suggested by fractional linear polarization measurements that quantify magnetic randomness. Specifically, the expression for fractional polarization $\alpha$ is (Wardle et al. 1994; Wardle 2013)

$$
\alpha=\frac{3 \iota^{2} \sin ^{2} \theta^{\prime}}{2+3 \iota^{2} \sin ^{2} \theta^{\prime}},
$$

where $\iota$ is the ratio of the uniform over random magnetic field strengths, and $\theta^{\prime}$ is the angle between jet and observer in the rest frame of the jet material. Using such measurements, Gómez et al. (2008) showed that the magnetic field is indeed quite disordered $(\alpha \sim 5 \%)$ in the central spine region of the jets (and more ordered at the edges with $\alpha \sim 15 \%-25 \%$ ).

One is then faced with the question of what mechanism is responsible for launching these magnetic loops and for creating the disordered environment they reside in. A particularly interesting observation is that the randomness in the magnetic field decreases as we move outward along the jet (Wardle 2013), from an $\alpha$ of zero to a few percent near the base of the jets to tens of percent at a few tens of beam widths away (Pollack et al. 2003; Lister \& Homan 2005; Gómez et al. 2008), a trend that appears inconsistent with randomness induced by jet flow instability. ITV turbulence provides an interesting alternative, with the benefit of being already present in the
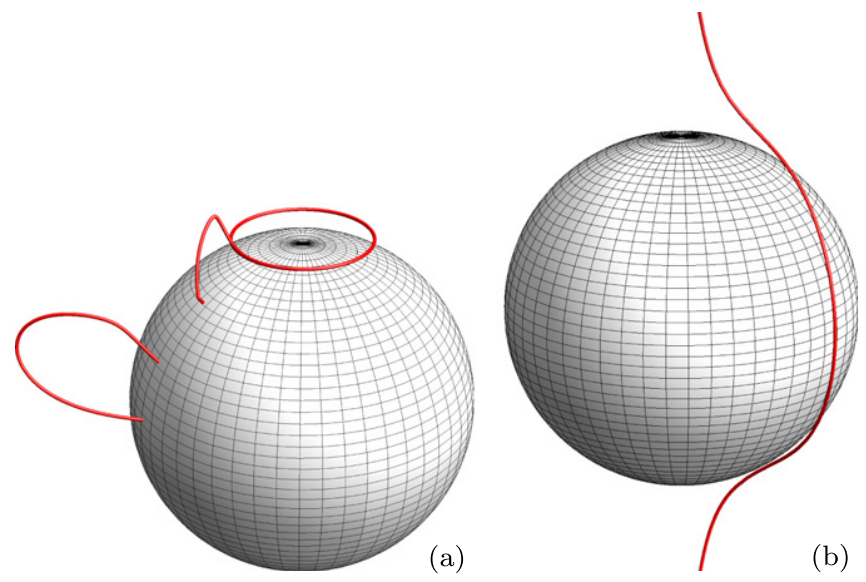

Figure 7. (a) A couple of arbitrarily chosen magnetic field lines (red) for the Wald solution perturbed by a $\Phi_{0}$ given by Equation (30) $(l=10)$. The gray sphere represents the black hole horizon. The preloops can become properly closed (if not already so) through magnetic reconnection later on within the jet. Note that although these individual magnetic field lines are not axisymmetric, there are others that are simple shifts of them in the azimuthal direction, so the magnetic field as a whole is axisymmetric. (b) A similar field line for the unperturbed Wald solution. The field line does not penetrate the horizon and does not form a preloop.

central engine. Aside from the turbulence in the electromagnetic field, because ITV turns up and down the energy extraction efficiency, it has the innate ability to produce disturbances in the jet material's radial velocity, encouraging the plasma flow to also take up traits of turbulence right from the beginning. The joint electromagnetic and fluid turbulences not only provide a possible explanation for the microvariability seen in jet emissions (randomness in the magnetic field translates directly into that of the synchrotron radiation), they are also beneficial for the random occurrences of magnetic reconnection (i.e., they increase the rate of reconnection; see Lazarian \& Vishniac 1999), which would help birth field line loops. ITV perturbations to the magnetic field also fragment otherwise smooth or straight magnetic field lines, bending them into preloops to be swept down the jet, so that the reconnection results are more likely to be loops rather than redirected open lines. Figure 7 depicts a couple of arbitrary magnetic field lines of a Wald solution perturbed by a toy $\Phi_{0}$ as given by Equation (30) (with $l=10$ ). As more loops are formed and subsequently sheared farther down stream, the magnetic field becomes more ordered into the poloidal direction, producing the aforementioned trend of decreasing randomness. Furthermore, this process occurs where energy extraction is the most efficient, so it is not unreasonable that the resulting loops are abundant in the more energetic spine of the jets. In contrast, if loops are produced by kinetic instabilities, for example, they would congregate near jet boundaries.

Finally, notice that in Figure 4(a), the perturbed field lines are not symmetric against the equatorial plane, indicating that ITV would likely feed energy into the two jets asymmetrically, even causing jets to become intrinsically one-sided given the right conditions. Observationally, one-sided or highly asymmetric jets are not rare (see Begelman et al. (1984) and in particular Spencer et al. (2001) and Russell et al. (2007) for Cygnus X-1). Although relativistic beaming may help explain the faintness of a mirror jet in some cases, some level of intrinsic asymmetry can not be ruled out (see Section II A 4 of Begelman et al. 1984). For example, interleaving regions of 


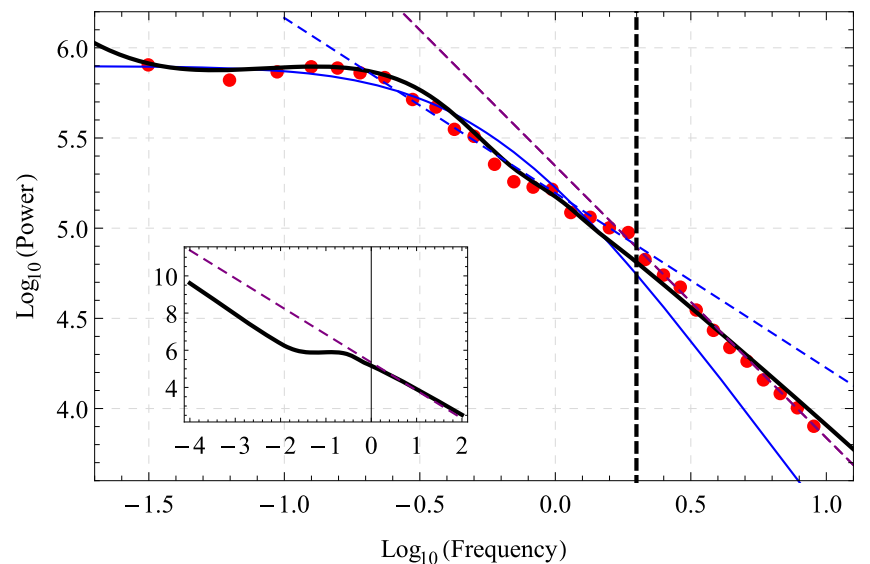

Figure 8. Red dots are the microvariation PSD data for Cygnus X-1 presented in Figure 2 of Uttley \& McHardy (2001). The black dashed vertical curve marks out the boundary between two "phases." The blue and purple dashed lines are local linear fits to segments of data close to the boundary, with slopes $\approx-1$ and $\approx-1.5$, respectively. The thin blue curve is a fit using a shot-noise model with a power-law decay function of power $p=6.26$. The thick black curve is a fit with the new hot-band model having a leakage profile (38) and a red underlying bombardment spectral density. The inset is a zoom-out of the thick black curve (and the purple dashed line for reference), showing that it returns to a power law at very low frequencies. In this figure, the frequency is in units of $\mathrm{Hz}$, and the power is in units of counts ${ }^{2} \mathrm{~s}^{-2} \mathrm{~Hz}^{-1}$.

emission in jets on alternative sides have been seen in a number of sources, suggesting a flip-flopping of an intrinsically onesided jet (Rudnick \& Edgar 1984).

\subsection{Manifestation in Disk Emission}

Let us now turn to examine the detailed shape of the microvariation PSD associated with X-ray observations. We will formulate our arguments within the context of disk- or corona-based inverse-Compton scattering (Bregman 1990), but note that jet emission may also contribute (Grandi \& Palumbo 2004), and our discussion can be adapted to that case as well. For concreteness, we take the very clean data obtained for Cygnus X-1 and presented in Figure 2 of Uttley \& McHardy (2001), which we partially reproduce (we only take their first flux quartile because the fourth quartile curve is similar, and we do not need the error bars that are very small) in Figure 8. The PSD exhibits a rich structure. For example, there appears to be a break in the data at around $\omega=2 \mathrm{~Hz}$, marked out with the black dashed vertical line in Figure 8. The points to the right have a steeper linear fit than the points immediately to the left, with slopes of $s_{r}=-1.51$ and $s_{l}=-0.97$, respectively. And more importantly, we will have to find a process to explain the PSD's white-turning-into-red shape (van der Klis 1995) to the left of the dashed line.

A leading explanation is the shot-noise model of Terrell (1972), which we will describe in a little detail because we will be using essentially the same mathematics. In this model, a white noise background PSD is produced by uncorrelated shots occurring within the disk or corona, which then decay away over time. One possible physical realization of the shots is spontaneously arising flares (e.g., the magnetic flares of Poutanen \& Fabian 1999) in the corona that die away gradually. The effect of the decay is that it is a slower process (as compared to the instantaneous leading edge of the shots) that smears out the higher-frequency components of the shots, resulting in a red/pink PSD for the emitted X-ray radiation at
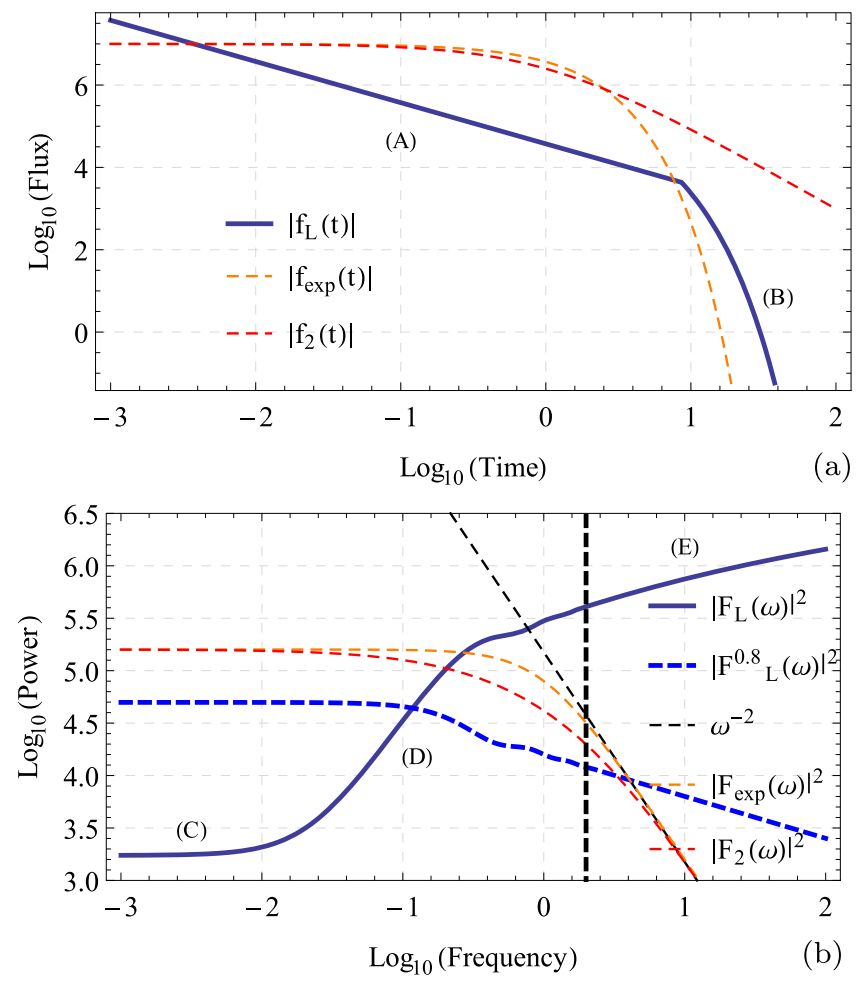

Figure 9. (a) The leakage/decay profile $f(t)$ in the time domain (see Figure 1 in Kazanas et al. 1997) for the leakage flux $f_{\mathrm{L}}(t)$ and the decays $f_{\exp }(t)$ (used in the shot-noise model of Terrell 1972) and power law $f_{2}(t)$. For $f_{\mathrm{L}}(t)$, the segments (A) and (B) correspond to the top and bottom lines in Equation (38). (b) The frequency domain leakage profile $\left|F_{\mathrm{L}}(\omega)\right|^{2}$ and its decay counterparts $\left|F_{\exp }(\omega)\right|^{2}$ and $\left|F_{2}(\omega)\right|^{2}$. The vertical dashed black line corresponds to the one in Figure 8. For $\left|F_{\mathrm{L}}(\omega)\right|^{2}$, the three segments (C), (D), and (E) correspond to the three pieces with different slopes in the inset of Figure 8. When the power index in Equation (38) is increased from -1 , as in the case of $\left|F_{\mathrm{L}}^{0.8}(\omega)\right|^{2}$, for which the index is changed to -0.8 , the profile becomes similar to the decays. For the decays, the exponential decay profile $\left|F_{\exp }(\omega)\right|^{2}$ has a flat plateau at low frequencies and a fixed slope of -2 at high frequencies (see the black dashed fitting line). Power-law decay profiles (red dashed curve) share the same general shape and the same slope of -2 at high frequencies, but they can have a smoother transition region. For both figures, the vertical axes are shifted arbitrarily so all curves fit into the same plot.

higher frequencies, while leaving the lower frequencies untouched and white. Mathematically, let the decay profile of a flare occurring at $t=0$ be modeled by some $f(t)$ : then its effect on the PSD is accounted for by a temporal convolution of $f(t)$ into the original flaring time series (a train of delta functions), which translates into a frequency space multiplication by $F(\omega)$, the Fourier transform of $f(t)$. So the overall PSD is multiplied by $|F(\omega)|^{2}$. In Terrell (1972), a decay is "arbitrarily" chosen to be of an exponential form, $f_{\exp }=\exp (-\lambda t) \Theta(t)$, where $\Theta$ is the Heaviside step function. We see that its Fourier transform $F_{\exp }=1 /[\sqrt{2 \pi}(\lambda-i \omega)]$ naturally provides the desired flat plateau in $\left|F_{\text {exp }}\right|^{2}$ at lower frequencies (see Figure 9(b)), so the choice is not entirely arbitrary. Nevertheless, we note that other decay profiles, with, for example, $f_{p}(t)=(t+1)^{-p} \Theta(t)$, have the same general $|F|^{2}$ shape in the frequency domain, but possibly more smooth transition regions (see Figure 9(b)). With all of these decay profiles, however, the slope of $|F|^{2}$ at high frequencies is fixed at -2 , quite different from the $s_{l} \approx-1$ and $s_{r} \approx-1.5$ values. This discrepancy is cited as the major shortcoming of the shotnoise model. By using power-law generalizations, one can trick 
the model to produce better fits to the data by placing the wider and smoother transition regions of $|F|^{2}$, instead of the steady, steeply sloped region, over the observational data. The best fit (to data on the left of the dashed line only; fitting to all data leads to worse-looking results) is produced with $p=6.26$ (the solid, thin blue curve in Figure 8). With this generalization, we achieve a decent fit for the data points at low frequencies, but the slope is unsurprisingly too high at higher frequencies.

Alternative generalizations introducing a wide range of different decay timescales (Lehto 1989; Lochner et al. 1991) can possibly achieve much better fits, as one would expect for an increased number of available tunable parameters, but they would also have a flat region extending all the way into extremely low frequencies. In contrast, it has been observed that (at least for galactic X-ray sources) a power-law PSD resumes at frequencies much lower than the critical value at which transition from white to red noises occurs in Figure 8, as possibly hinted at by the uptick in the left-most data point in Figure 8 (the extent of these data into low $\omega$ values is limited by observation-duration constraints), so the flat region is only a shoulder in the broader spectrum (Kazanas et al. 1997). In addition, the standard shot-noise model (and some other models such as self-organized criticality) is also incompatible with the flux dependence of the variation amplitude discussed in Section 5.1 (Gaskell 2004). We now introduce an alternative proposal that achieves a tighter fit (with only one additional physically motivated adjustable parameter) and a return to a power law at very low frequencies.

Specifically, energy extracted from the black hole can travel along magnetic field lines in the form of Alfvén waves ${ }^{7}$ (for rapidly spinning black holes, the disk can get very close to the horizon and so penetrate inside the magnetosphere, where plasma waves are allowed to propagate). Since the field lines thread through the accretion disk, energy flows would bombard the disk and corona, heating them up to create hot bands. The turbulent ITV would then cause not only a changing energyextraction rate at the source, but also a focusing and defocusing of Alfvén waves by altering how tightly the field lines bundle. The consequence is that the bombardment takes on a stochastic appearance, with a PSD consistent with the electromagnetic turbulence discussed previously. For this section, we take it to be a Kolmogorov $\omega^{-5 / 3}$ power law (but noting that making this more flexible could result in even better fits to the data). Note that the average high-frequency slopes of $s_{r} \approx-1.5$ for Cygnux X-1 and $\approx-1.55$ in similar PSDs for AGNs measured by Lawrence \& Papadakis (1993) are inconsistent with either the standard shot-noise model $(-2)$ or the traditional flicker noise $(-1)$, but are close to $-5 / 3$.

The hot bands can then serve as sources for soft photons and hot electrons, replacing the accretion shocks in the inhomogeneous hot cloud model of Kazanas et al. (1997). The hot cloud model is constructed to explain the observed time lags between hard and soft photons, as well as the power spectrum shape. Specifically, Kazanas et al. (1997) propose a Comptonizationbased emission mechanism that requires the existence of large

\footnotetext{
The waves propagating inside a force-free magnetosphere abutting the thin vacuum layer can be divided into two categories: the fast magnetosonic waves and the Alfvén waves. A feature worth noting is that the Alfvén waves are good candidates for carrying energy out of the central engine region (see Brennan et al. 2013; Zhang et al. 2015 for an exact analytical solution that does so efficiently) because their propagation is regulated to be along magnetic field lines, rather than scattering randomly. Fast magnetosonic waves, on the other hand, propagate more like vacuum electromagnetic waves.
}

hot clouds with most of the energy at the outer boundary (in order to match the frequency dependence of the lags). This model has been criticized on the grounds that such a peculiar energy distribution is difficult to produce physically if one assumes that the energy sponsor for the clouds is internal to the disk. However, with our external energy source, it is natural for hot bands or clouds to be more energetic on their boundaries that are more exposed to bombardments. Since it takes time for $\mathrm{X}$-ray photons to escape the hot cloud (in particular, hard photons need to experience more scattering inside the cloud to pick up energy and thus emerge later), a leakage profile needs to be overlaid on the energy bombardment profile to produce the final radiation PSD, and the mathematical details become similar to the shot-noise model (convolution in the time domain translating into multiplications in the frequency domain), but the underlying white shot-noise curve (also assumed for the original hot cloud model; see below) is replaced by a red $\omega^{-5 / 3}$ power law, and a leakage profile replaces the decay profile.

For the photon leakage, simulations in Kazanas et al. (1997; see Figure 1 therein) produced results mostly hugging the $f_{\mathrm{L}}(t)=t^{-1} \Theta(t)$ profile at small $t$. At larger $t$, the simulations exhibit a transition from the power law into an exponential cutoff that declines much faster. We denote the time when this occurs as $C_{1}$, so the leakage profile is

$$
f_{\mathrm{L}}(t)= \begin{cases}A t^{-1}, & 0 \leqslant t<C_{1}, \\ A t^{-1} e^{-\lambda\left(t-C_{1}\right)}, & t \geqslant C_{1} .\end{cases}
$$

In the frequency domain, we then have

$$
\begin{gathered}
F_{\mathrm{L}}(\omega)=A^{\prime}\left\{\left[\log (B \omega)+\gamma-\operatorname{Ci}\left(B \omega C_{1}^{\prime}\right)-i \operatorname{Si}\left(B \omega C_{1}^{\prime}\right)\right]\right. \\
\left.+e^{C_{1}^{\prime} \lambda^{\prime}}\left[\operatorname{Ei}\left(\left(i B \omega-\lambda^{\prime}\right) C_{1}^{\prime}\right)-\operatorname{Ei}\left(\left(i B \omega-\lambda^{\prime}\right) C_{2}^{\prime}\right)\right]\right\}
\end{gathered}
$$

where $\gamma$ is Euler's constant, while $\mathrm{Si}, \mathrm{Ci}$, and $\mathrm{Ei}$ are the sine, cosine, and exponential integral functions. Note we have introduced a $B$ factor multiplying onto $\omega$, which makes it easier to move $F_{\mathrm{L}}(\omega)$ across frequencies by hand to zero in on an initial guess for the fitting. This corresponds to a rescaling of $t$ by multiplying it with $1 / B$, resulting in the relationships $\lambda=\lambda^{\prime} / B$ and $C_{1}=B C_{1}^{\prime}$. We have also introduced an overall cutoff at $C_{2}^{\prime} \gg C_{1}^{\prime}$ (i.e., $f(t)=0$ exactly when $t>B C_{2}^{\prime}$ ), which helps with the Fourier transformation routine but has no physical effects because the exponential suppression factor has already ensured that $f_{\mathrm{L}}(t) \approx 0$ at $t=B C_{2}^{\prime}$, so $C_{2}^{\prime}$ can be chosen to have any arbitrary large value. Adjusting the remaining parameters in Equation (39) via a simple, selective (accepting a step only when it leads to an improvement), random-walk procedure produces the best-fit PSD as the thick black curve in Figure 8, corresponding to the parameter values (leaving out the unimportant overall amplitude $A^{\prime}$, which is degenerate with the amplitude of the underlying bombardment profile)

$$
B \approx 15.0, \quad \lambda^{\prime} \approx 4.98, \quad C_{1}^{\prime} \approx 0.577,
$$

translating into

$$
\lambda \approx 0.333, \quad C_{1} \approx 8.63 s .
$$

The $f_{\mathrm{L}}(t)$ and corresponding $F_{\mathrm{L}}(\omega)$ profiles with these parameters are shown in Figure 9. We note in particular that the $C_{1}$ value in Equation (41) is consistent with the results of 
Kazanas et al. (1997), obtained using realistic astrophysical parameters for Cygnus X-1.

We reiterate that although our leakage profile is similar to those from the simulations of Kazanas et al. (1997), the power to $t$ in our $f_{\mathrm{L}}(t)$ is precisely -1 , which creates a more interesting profile for $F_{\mathrm{L}}(\omega)$, as shown in Figure 9(b). In the $\log -\log$ plots, the profiles of $\left|F_{\mathrm{L}}(\omega)\right|^{2}$ and the bombardment profile of $\omega^{-5 / 3}$ (a straight line with slope $-5 / 3$ ) combine by addition, so the three segments marked (C), (D), and (E) in Figure 9(b) create three regimes of different slopes (see the inset in Figure 8). Piece (D) neutralizes the bombardment slope $-5 / 3$, creating the flat shoulder, while segments (C) and $(\mathrm{E})$, being much flatter themselves, have less impact. In contrast, Kazanas et al. (1997) had leakage profiles whose power indices are close to but a little more positive than -1 , resulting in simpler $\left|F_{\mathrm{L}}(\omega)\right|^{2}$ profiles (see the dashed blue curve in Figure 9(b)), which in fact more closely resemble the decay profiles like $\left|F_{\exp }(\omega)\right|^{2}$. Therefore, those leakage profiles need to be overlaid on a white-shot-noise profile in order to produce a white-noise plateau, just as in the shot-noise model. In other words, in either the shot-noise model or the original hot cloud model, a white underlying shot noise has to be present in the first place, which is responsible for the plateau, and is masked at higher frequencies by shorter timescale phenomena related to the evolution of each individual shot. At the other end of the spectrum, there is no natural way to mask the white noise at very low frequencies, so in order to return to a power law there, one has to invoke new and separate mechanisms (such as accretion-rate fluctuations that are due to variations in the viscosity parameter (Lyubarskii 1997)). Our proposal works quite differently. The bombardment profile is a red noise, and nothing is intrinsically white, so the white-noise shoulder is necessarily approximate and transient, and the morphology of the entire PSD can be accounted for with a single mechanism.

\section{CONCLUSION}

We caution that most, if not all, of the observational phenomena discussed above admit alternative explanations, mostly involving more complicated disk and corona dynamics (e.g., the existence of many different decay scales, fractal chain of flares, and self-organized criticality in disk fluctuations), and, indeed, multiple mechanisms are likely at work. The particular proposal introduced here has some features that we think are desirable and make it a useful candidate. First of all, ITV is an integral part of the fundamental energy process and propagates at the speed of light, so it is capable of producing the observed large variations that evolve on a light-crossing (and not viscous) timescale (Gaskell \& Klimek 2003). It is also common across different celestial objects with mass scales spanning decades. Specifically, the ITV dynamics is largely governed by the properties of the black holes, whose simplicity (as exemplified by, e.g., no-hair theorems (Chrusciel 1994; Heusler 1998) when the holes are in isolation) leads to simpler scaling laws (an example is the numerical computation of gravitational waveforms for binary black hole coalescences, where only one simulation is needed, and then the resulting waveform applies to different mass scales after a simple rescaling of the axes), so ITV in AGNs is not expected to qualitatively differ from that in microquasars and quantitatively should scale proportionally according to black hole mass. One commonality that jumps out immediately is that, although we have taken Cygnus X-1 as an example in Section 5.3, the PSDs for AGNs share remarkably similar features (see, for example, Figure 1 of Romero et al. (2002) for blazar 0208-512). In addition, the characteristic variation timescale indeed scales with the black hole mass (Edelson \& Nandra 1999; Gaskell \& Klimek 2003).

Although we have initiated our ITV discussions in the context of evading the Meissner effect and thus have concentrated on rapidly spinning black holes, we note that ITV may nevertheless be present around more moderately spinning holes as well. So long as it increases the horizon flux as compared to the otherwise stationary background (not yet known at the present time), the discussions in Section 5 still apply. It is, however, likely that ITV is less violent when teamed with lower spins. Tchekhovskoy et al. (2010) argues for higher spin in radio-loud sources, and, on the other hand, the variabilities of the radio-loud and radio-quiet objects tend to be more violent and quiescent, respectively, especially on the shorter timescales (Bregman 1990), which would at first sight, at least, be consistent with more violent ITV for sources powered by more rapidly spinning holes.

Finally, due to the difficulty in quantitatively solving joint evolution systems containing electromagnetic, general relativistic, fluid dynamic, and radiation microphysical ingredients, some of the discussions are limited to a vastly simplified and heuristic level. We hope that further studies in the future, especially through numerical simulations, will provide more concrete predictions to compare with data and perhaps reveal more intriguing and unexpected properties of electromagnetism near rapidly spinning black holes. In particular, the ITV turbulence needs to be verified numerically and its energy spectrum better characterized, through targeted studies with sufficient accuracy to resolve small-scale features and the ability to distinguish randomness caused by ITV and numerical errors, so overzealous evolution-stabilization measures are not triggered to artificially suppress ITV. The numerical code may also need to be able to account for the important structures surrounding a black hole in an astrophysical setting, to see if energy flows reflected off, for example, the interstellar medium or the ion-supported or dusty torus result in a black hole bomblike condition (see Press \& Teukolsky (1972), but with the BZ process replacing superradiance and the mirror becoming possibly leaky and only partially enclosing), which would benefit the self-sustainability of ITV.

This work is supported by the National Natural Science Foundation of China Grants 11443008 and 11503003, the Fundamental Research Funds for the Central Universities Grant 2015KJJCB06, and a Returned Overseas Chinese Scholars Foundation grant.

\section{REFERENCES}

Antonucci, R. 1993, ARA\&A, 31, 473

Aretakis, S. 2012, arXiv:1206.6598

Aretakis, S. 2013, PhRvD, 87, 084052

Begelman, M. C., Blandford, R. D., \& Rees, M. J. 1984, RvMP, 56, 255

Bicak, J., \& Dvorak, L. 1976, GReGr, 7, 959

Bicák, J., \& Dvorák, L. 1980, PhRvD, 22, 2933

Bicak, J., \& Janis, V. 1985, MNRAS, 212, 899

Bicak, J., \& Karas, V. 1989, in Proc. of the 5th Marcel Grossman Meeting on General Relativity, 1199, ed. D. G. Blair, \& M. K. Buckingham (Singapore: World Scientific)

Bičák, J., \& Hejda, F. 2015, arXiv:1510.01911

Bicak, J., Karas, V., \& Ledvinka, T. 2006, arXiv:astro-ph/0610841, [IAU Symp. 238, 139(2007)] 
Bičák, J., Karas, V., \& Ledvinka, T. 2007, in IAU Symp. 238, Black Holes from Stars to Galaxies-Across the Range of Masses, ed. V. Karas, \& G. Matt (Cambridge: Cambridge Univ. Press), 139

Blandford, R. D., \& Königl, A. 1979, ApJ, 232, 34

Blandford, R. D., \& Payne, D. G. 1982, MNRAS, 199, 883

Blandford, R. D., \& Znajek, R. L. 1977, MNRAS, 179, 433

Brandenburg, A., Kahniashvili, T., \& Tevzadze, A. G. 2015, PhRvL, 114, 075001

Bregman, J. N. 1990, A\&ARv, 2, 125

Brennan, T. D., Gralla, S. E., \& Jacobson, T. 2013, CQGra, 30, 195012

Chamblin, A., Emparan, R., \& Gibbons, G. W. 1998, PhRvD, 58, 084009

Cho, J. 2005, ApJ, 621, 324

Chrusciel, P. T. 1994, Contemp. Math., 170, 23

Contopoulos, I., Kazanas, D., \& Papadopoulos, D. B. 2013, ApJ, 765, 113

Degrange, B., Superina, G., Giebels, B., \& Volpe, F. 2008, Blazar Variability Across the Electromagnetic Spectrum, 16

Dhalla, S. M., Webb, J. R., Bhatta, G., \& Pollock, J. T. 2010, JSARA, 4, 7

Edelson, R., Mushotzky, R., Vaughan, S., et al. 2013, ApJ, 766, 16

Edelson, R., \& Nandra, K. 1999, ApJ, 514, 682

Elvis, M., Risaliti, G., \& Zamorani, G. 2002, ApJL, 565, L75

Gammie, C. F., Shapiro, S. L., \& McKinney, J. C. 2004, ApJ, 602, 312

Gaskell, C. M. 2004, ApJL, 612, L21

Gaskell, C. M., \& Klimek, E. S. 2003, A\&AT, 22, 661

Gaur, H., Gupta, A. C., Lachowicz, P., \& Wiita, P. J. 2010, ApJ, 718, 279

Gibbons, G. W., Mujtaba, A. H., \& Pope, C. N. 2013, CQGra, 30, 125008

Giebels, B., \& Degrange, B. 2009, A\&A, 503, 797

Goldreich, P., \& Julian, W. H. 1969, ApJ, 157, 869

Goldreich, P., \& Sridhar, S. 1995, ApJ, 438, 763

Gómez, J. L., Marscher, A. P., Jorstad, S. G., Agudo, I., \& Roca-Sogorb, M. 2008, ApJL, 681, L69

Gou, L., McClintock, J. E., Reid, M. J., et al. 2011, ApJ, 742, 85

Grandi, P., \& Palumbo, G. G. C. 2004, Sci, 306, 998

Gupta, A. C., Fan, J. H., Bai, J. M., \& Wagner, S. J. 2008, AJ, 135, 1384

Hardee, P. 2008, JPhCS, 131, 012052

Hejda, F., \& Bicak, J. 2014, in WDS'14 Proc. Contributed Papers-Physics, ed. J. Šafránková, \& J. Pavlů, 48

Heusler, M. 1998, LRR, 1, 6

Hook, I. M., McMahon, R. G., Boyle, B. J., \& Irwin, M. J. 1994, MNRAS, 268, 305

Hughes, P. A. 2005, ApJ, 621, 635

Hughes, P. A., Aller, M. F., \& Aller, H. D. 2011, ApJ, 735, 81

Jacobson, T. 2011, CQGra, 28, 187001

Jones, T. W. 1988, ApJ, 332, 678

Junor, W., Biretta, J. A., \& Livio, M. 1999, Natur, 401, 891

Karas, V., \& Budinova 2000, PhyS, 61, 253

Karas, V., \& Vokrouhlický, D. 1991, JMP, 32, 714

Kazanas, D., Hua, X.-M., \& Titarchuk, L. 1997, ApJ, 480, 735

King, A. R. 1977, MPCPS, 81, 149

King, A. R., Lasota, J. P., \& Kundt, W. 1975, PhRvD, 12, 3037

Kinnersley, W. 1969, JMP, 10, 1195

Lawrence, A., \& Papadakis, I. 1993, ApJL, 414, L85

Lazarian, A., \& Vishniac, E. T. 1999, ApJ, 517, 700

Lehto, H. J. 1989, in ESA Special Publication 296, Two Topics in X-ray Astronomy, Vol. 1, 2, ed. J. Hunt, \& B. Battrick, 499

Lister, M. L., \& Homan, D. C. 2005, AJ, 130, 1389

Lochner, J. C., Swank, J. H., \& Szymkowiak, A. E. 1991, ApJ, 376, 295

Lucietti, J., \& Reall, H. S. 2012, PhRvD, 86, 104030

Lyubarskii, Y. E. 1997, MNRAS, 292, 679

Lyutyj, V. M., \& Oknyanskij, V. L. 1987, AZh, 64, 465
McClintock, J. E., Narayan, R., \& Steiner, J. F. 2013, arXiv:1303.1583 McClintock, J. E., Shafee, R., Narayan, R., et al. 2006, ApJ, 652, 518 McKinney, J. C., \& Gammie, C. F. 2004, ApJ, 611, 977

McKinney, J. C., Tchekhovskoy, A., \& Blandford, R. D. 2012, MNRAS, 423, 3083

Miller, J. M., Fabian, A. C., Wijnands, R., et al. 2002, ApJL, 570, L69

Miniutti, G., Fabian, A. C., \& Miller, J. M. 2004, MNRAS, 351, 466

Mocanu, G. R., \& Sándor, B. 2012, Ap\&SS, 342, 147

Narayan, R., \& McClintock, J. E. 2012, MNRAS, 419, L69

Pan, Z., \& Yu, C. 2015, arXiv:1511.07925

Penna, R. F. 2014, PhRvD, 89, 104057

Penna, R. F., Narayan, R., \& Sa̧dowski, A. 2013, MNRAS, 436, 3741

Pollack, L. K., Taylor, G. B., \& Zavala, R. T. 2003, ApJ, 589, 733

Poutanen, J., \& Fabian, A. C. 1999, MNRAS, 306, L31

Press, W. H., \& Teukolsky, S. A. 1972, Natur, 238, 211

Rees, M. J., Begelman, M. C., Blandford, R. D., \& Phinney, E. S. 1982, Natur, 295,17

Reynolds, C. S. 2013, CQGra, 30, 244004

Romero, G. E., Cellone, S. A., Combi, J. A., \& Andruchow, I. 2002, A\&A, 390,431

Rosen, A., Hardee, P. E., Clarke, D. A., \& Johnson, A. 1999, ApJ, 510, 136

Rudnick, L., \& Edgar, B. K. 1984, ApJ, 279, 74

Russell, D. M., Fender, R. P., Gallo, E., \& Kaiser, C. R. 2007, MNRAS, 376,1341

Sasada, M., Uemura, M., Arai, A., et al. 2008, PASJ, 60, L37

Spencer, R., de La Force, C., Stirling, A., et al. 2001, ApSSS, 276, 255

Steiner, J. F., McClintock, J. E., \& Narayan, R. 2013, ApJ, 762, 104

Superina, G., \& Degrange, B. 2008, PoS, BLAZARS2008, 066

Takamori, Y., Nakao, K.-I., Ishihara, H., Kimura, M., \& Yoo, C.-M. 2011, MNRAS, 412, 2417

Tchekhovskoy, A., McKinney, J. C., \& Narayan, R. 2012, JPhCS, 372, 012040

Tchekhovskoy, A., Narayan, R., \& McKinney, J. C. 2010, ApJ, 711, 50

Terrell, N. J., Jr. 1972, ApJL, 174, L35

Teukolsky, S. 1973, ApJ, 185, 635

Teukolsky, S., \& Press, W. 1974, ApJ, 193, 443

Thorne, K. S. 1974, ApJ, 191, 507

Thorne, K. S., Price, R. H., \& MacDonald, D. A. 1986, Black Holes: The Membrane Paradigm (1st ed.; New Haven, CT and London: Yale Univ. Press)

Urry, C. M., \& Padovani, P. 1995, PASP, 107, 803

Uttley, P., \& McHardy, I. M. 2001, MNRAS, 323, L26

Uttley, P., McHardy, I. M., \& Vaughan, S. 2005, MNRAS, 359, 345

van der Klis, M. 1995, in X-ray Binaries, ed. W. H. G. Lewin (Cambridge: Cambridge Univ. Press), 252

Vaughan, S., Edelson, R., Warwick, R. S., \& Uttley, P. 2003, MNRAS, 345,1271

Wald, R. M. 1974, PhRvD, 10, 1680

Wang, J.-M., Chen, Y.-M., Ho, L. C., \& McLure, R. J. 2006, ApJL, 642, L111

Wardle, J. F. C. 2013, in European Physical Journal Web of Conf. 61, 6001

Wardle, J. F. C., Cawthorne, T. V., Roberts, D. H., \& Brown, L. F. 1994, ApJ, 437, 122

Wold, M., Brotherton, M. S., \& Shang, Z. 2007, MNRAS, 375, 989

Yang, H., Zhang, F., Green, S. R., \& Lehner, L. 2015, PhRvD, 91, 084007

Yang, H., Zimmerman, A., Zenginolu, A., et al. 2013, PhRvD, 88, 044047

Zhang, F., Brink, J., Szilágyi, B., \& Lovelace, G. 2012, PhRvD, 86, 084020

Zhang, F., McWilliams, S. T., \& Pfeiffer, H. P. 2015, PhRvD, 92, 024049

Zrake, J. 2014, ApJL, 794, L26

Zrake, J., \& East, W. E. 2015, arXiv:1509.00461 